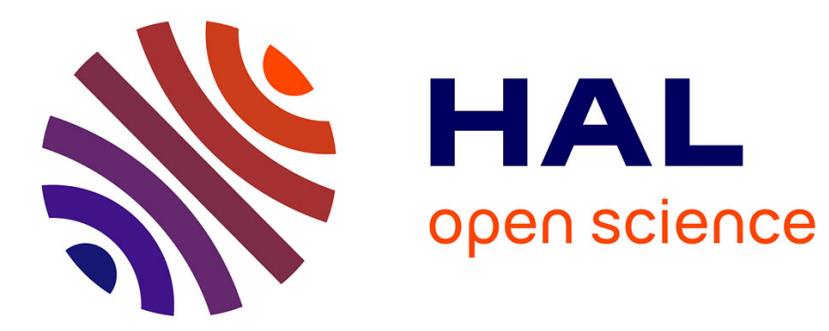

\title{
Distribution radiale de la densité électronique et de la densité des atomes excités dans une colonne de plasma produite par une onde de surface
}

M. Moisan, R. Pantel, André Ricard, V.M.M. Glaude, P. Leprince, W.P. Allis

\section{- To cite this version:}

M. Moisan, R. Pantel, André Ricard, V.M.M. Glaude, P. Leprince, et al.. Distribution radiale de la densité électronique et de la densité des atomes excités dans une colonne de plasma produite par une onde de surface. Revue de Physique Appliquée, 1980, 15 (8), pp.1383-1397. 10.1051/rphysap:019800015080138300 . jpa-00244862

HAL Id: jpa-00244862 https://hal.science/jpa-00244862

Submitted on 1 Jan 1980

HAL is a multi-disciplinary open access archive for the deposit and dissemination of scientific research documents, whether they are published or not. The documents may come from teaching and research institutions in France or abroad, or from public or private research centers.
L'archive ouverte pluridisciplinaire HAL, est destinée au dépôt et à la diffusion de documents scientifiques de niveau recherche, publiés ou non, émanant des établissements d'enseignement et de recherche français ou étrangers, des laboratoires publics ou privés. 


\title{
Distribution radiale de la densité électronique et de la densité des atomes excités dans une colonne de plasma produite par une onde de surface
}

\author{
M. Moisan, R. Pantel, A. Ricard (*), V. M. M. Glaude, P. Leprince (*) et W. P. Allis (**) \\ Département de Physique, Université de Montréal, C.P. 6128, succursale A, Montréal, Québec H3C 3J7, Canada
}

(Reçu le 7 février 1980, révisé le 3 avril 1980, accepté le 16 avril 1980)

\begin{abstract}
Résumé. - On détermine expérimentalement la distribution radiale de la densité des atomes excités dans une colonne de plasma d'argon produite par une onde de surface. Ces mesures sont réalisées en enregistrant directement par prospection radiale, soit l'émission des atomes excités, soit l'absorption que ces atomes produisent sur un rayonnement incident de référence. Suivant la pression du gaz (entre 50 et 350 mtorr) et la fréquence de l'onde de surface, les distributions radiales observées présentent une forme presque plate ou, au contraire, montrent un creux plus ou moins profond sur l'axe avec un maximum relatif situé au voisinage de la paroi. On explique ce comportement pour un niveau radiatif en montrant théoriquement que sa densité doit être proportionnelle à $n(r) E^{2}(r)$ où $r$ est la position radiale, $n(r)$, la densité électronique et $E^{2}(r)$, l'intensité du champ électrique de l'onde de surface. La même dépendance apparaît théoriquement et expérimentalement pour la densité d'un niveau métastable, lorsque la destruction de ce niveau est principalement assurée par collisions avec des atomes neutres. La distribution radiale des électrons n'est pas mesurée mais calculée. Bien que la production des électrons dépende localement de la forme du champ électrique de l'onde de surface, dans la mesure où la diffusion ambipolaire est le principal mécanisme de pertes des particules chargées, la distribution radiale des électrons s'écarte assez peu d'une fonction de Bessel $J_{0}$.
\end{abstract}

\begin{abstract}
We have observed the radial density distribution of excited atoms in an argon plasma produced by a surface wave. The measurements are made by recording both emission and absorption at a given wavelength, as a function of radius. Depending on the neutral gas pressure (between 50 and 350 mtorr) and on the surface wave frequency, the radial distribution is either flat or has a minimum on the axis and a maximum near the tube wall. This behaviour is explained theoretically for radiative levels by showing that their population density should be proportional to $n(r) E^{2}(r)$ where $r$ is the radius, $n(r)$ is the electron density and $E^{2}(r)$ is the electric field strength of the surface wave. The same radial dependence occurs theoretically and experimentally for the density of a metastable level, provided its destruction mainly results from collisions with neutral atoms. The radial distribution of the electrons is known only through a calculation assuming ambipolar diffusion. With this assumption, the radial distribution of electrons can be well approximated by a $J_{0}$ Bessel function, though the electron production varies radially with the electric field intensity.
\end{abstract}

1. Introduction. - Des travaux récents ont montré la possibilité et les conditions adéquates pour produire de longues colonnes de plasma au moyen d'énergie hyperfréquence $(\mathrm{HF})$, grâce à la propagation d'une onde de surface $[1,2,3]$. Le diamètre de ces colonnes peut varier de $0,5 \mathrm{~mm}$ (capillaire) jusqu'à plusieurs dizaines de $\mathrm{mm}$. Ce plasma est produit de façon stable

$\left(^{*}\right)$ Laboratoire de Physique des Plasmas, Université Paris XI, 91405 Orsay, France.

(**) Physics Department, Massachusetts Institute of Technology, Cambridge 02139, Etats-Unis. dans un domaine de pression qui recouvre celui de la colonne positive d'une décharge électrique en courant continu, sauf dans le cas des plasmas de grand diamètre, où la pression maximale de fonctionnement semble moins élevée. On constate d'autre part qu'aux faibles pressions, ce plasma s'établit plus facilement que la décharge en courant continu. L'énergie HF se trouve transmise au plasma par une structure (Surfatron) située à l'extérieur du tube de plasma. Pour ces raisons et du fait de l'absence de stries, d'un taux de fluctuations de la densité électronique extrêmement faible, le plasma d'onde de surface pourrait s'avérer un substitut intéressant à la colonne positive 
classique. Une telle éventualité nécessite un examen détaillé des caractéristiques de ce plasma.

Une première étude comparative de la population des niveaux excités (métastables et résonnants) des atomes d'argon d'un plasma d'onde de surface avec celle d'un plasma de colonne positive, dans les mêmes conditions de diamètre de plasma et de pression de gaz, a déjà été réalisée [4]. La population de ces niveaux a été déterminée par absorption en visant perpendiculairement à l'axe du plasma, suivant un diamètre, en un point le long de la colonne. La densité ainsi obtenue pour chaque niveau est une densité moyenne le long de ce diamètre. De plus, la puissance HF ou le courant de décharge était toujours ajusté de telle sorte que l'on observe la saturation en densité des niveaux excités. Dans ces conditions, on a remarqué que sur une certaine plage de pression, la densité des atomes excités du plasma d'onde de surface est de deux à sept fois supérieure à la densité correspondante de la colonne positive.

Le présent travail a pour but de reprendre cette étude en effectuant directement une prospection radiale de l'émission et de l'absorption de certains niveaux excités du plasma d'onde de surface. Ces résultats sont comparés, à titre de référence, avec ceux que nous obtenons, par la même méthode, dans un plasma de colonne positive de même diamètre, pour des mêmes pressions de gaz. Enfin, pour simplifier la comparaison avec le modèle théorique, la puissance HF produisant l'onde de surface est ajustée de façon à ce que l'onde ne se réfléchisse pas sur les extrémités de la colonne, évitant ainsi l'apparition d'ondes stationnaires le long de la colonne.

Expérimentalement, nous avons examiné l'effet de la fréquence de l'onde et de la pression du gaz sur la distribution radiale des atomes excités, radiatif ou métastable (Sect. 3). Contrairement à la colonne positive, on observe dans un plasma d'onde de surface, une grande diversité de formes dans la distribution radiale de densité des atomes excités.

Un modèle théorique a été élaboré (Sect. 4) qui rend compte des principaux faits expérimentaux. Ce modèle prédit que la distribution radiale de densité des atomes radiatifs est proportionnelle à $n(r) E^{2}(r)$ où $r$ est la position radiale, $n(r)$, la densité électronique et $E^{2}(r)$, l'intensité du champ électrique de l'onde. La distribution $n(r)$ des électrons n'est pas obtenue expérimentalement, elle est calculée avec l'hypothèse que les électrons disparaissent principalement par diffusion ambipolaire vers les parois. Quant à la valeur du champ $E(r)$, elle peut s'obtenir des équations régissant l'onde de surface, pourvu que l'on tienne compte de l'inhomogénéité radiale due à la distribution $n(r)$ des électrons.

Dans la section 5, on compare les données expérimentales avec les valeurs calculées d'après le modèle théorique. Ce modèle comporte deux paramètres d'ajustement : $E(a) / E(0)$ et $n(a) / n(0)$, la valeur du champ électrique et de la densité électronique sur la paroi relativement à la valeur sur l'axe. On montre que ces valeurs d'ajustement sont compatibles avec les valeurs observées des paramètres de l'onde de surface.

Une certaine connaissance des caractéristiques de l'onde de surface étant nécessaire à la compréhension des phénomènes étudiés, nous en rappelons l'essentiel dans la section 2 qui suit.

2. Caractéristiques des ondes de surface. -2.1 MODÈLE DU PLASMA HOMOGÈNE. - On peut obtenir une description simple mais très satisfaisante de la propagation d'une onde de surface sur un plasma, en considérant ce dernier comme un milieu diélectrique possédant une permittivité dont la valeur, relativement à celle du vide, est [5] :

$$
\varepsilon_{\mathrm{p}}=1-\frac{\omega_{\mathrm{pe}}^{2}}{\omega(\omega+j v)}
$$

où $\omega_{\mathrm{pe}}=2 \pi f_{\mathrm{pe}}$ est la pulsation des électrons du plasma et est donnée par $\left(n e^{2} / m \varepsilon_{0}\right)^{1 / 2}, n$ étant la densité des électrons, $e$ et $m$, la charge et la masse de l'électron, $\varepsilon_{0}$ la permittivité du vide; $\omega=2 \pi f_{0}$ est la fréquence angulaire de l'onde et $v$, la fréquence de collisions électron-neutre pour le transfert de la quantité de mouvement.

Dans les conditions d'existence de l'onde de surface, la partie réelle de $\varepsilon_{\mathrm{p}}$ est négative.

L'excitateur et la colonne de plasma présentant une symétrie de révolution, on peut s'attendre à ce que l'onde possède la symétrie azimutale $(s=0$ où $s$ est le nombre d'onde azimutal). Nous l'avons vérifié en constatant que la phase de l'onde ne dépend pas de l'angle azimutal $\varphi$. Dans ces conditions, seule une onde TM $\left(E_{z}, E_{\mathrm{r}}, H_{\varphi}\right)$ peut exister [5]

Pour obtenir l'équation de dispersion de l'onde de surface, on écrit les expressions des composantes du champ électromagnétique dans les divers milieux (plasma, diélectrique, air, Fig. 1) en résolvant les

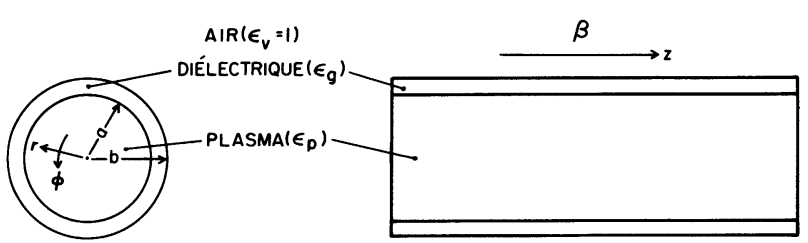

Fig. 1. - Géométrie de la colonne de plasma et orientation du vecteur d'onde $\beta$ de l'onde de surface.

[Geometry of the plasma column and direction of the surface wave vector $\beta$.]

équations de Maxwell. La loi de la continuité des champs $E_{z}$ et $H_{\varphi}$ aux interfaces permet d'obtenir un système de 4 équations à 4 inconnues qui ne peuvent être compatibles que si son déterminant est nul. Le calcul de ce déterminant permet d'obtenir la relation de dispersion : $\omega$ en fonction de $\beta$ avec $\omega_{\mathrm{pe}}=$ Cte 
( $\beta: 2 \pi / \lambda$, partie réelle du vecteur d'onde) ou la relation de phase : $\omega_{\mathrm{pe}}$ en fonction de $\beta$ avec $\omega=$ Cte. Cette dernière relation a été vérifiée expérimentalement à $360 \mathrm{MHz}$ [2]. La figure 2 permet d'obtenir les caractéristiques de l'onde de surface dans le cas où le plasma est créé par l'onde. L'interférogramme $2 \mathrm{C}$ montre que l'onde progressive est atténuée. On peut mesurer $\beta$ et $\alpha$ ( $\alpha$ partie imaginaire du vecteur d'onde) en fonction de la densité. L'intensité lumineuse globale, représentée en $2 \mathrm{~B}$, est proportionnelle à la densité électronique [6].

Sur la figure 3 , on a représenté les champs $E_{z}(r)$ et $E_{\mathrm{r}}(r)$ dans le plasma pour 2 valeurs du rapport $f_{\mathrm{pe}} / f_{0}$. On constate que le champ $E_{z}$ est beaucoup plus important que $E_{\mathrm{r}}$ et qu'il croît de l'axe vers les parois où il est maximum. L'expression des champs est donnée par:

$$
\begin{aligned}
& E_{z}(r)=A_{1} I_{0}\left(\sqrt{\beta^{2}-\beta_{0}^{2} \varepsilon_{\mathrm{p}}} r\right) \\
& E_{\mathrm{r}}(r)=A_{2} I_{1}\left(\sqrt{\beta^{2}-\beta_{0}^{2} \varepsilon_{\mathrm{p}}} r\right)
\end{aligned}
$$

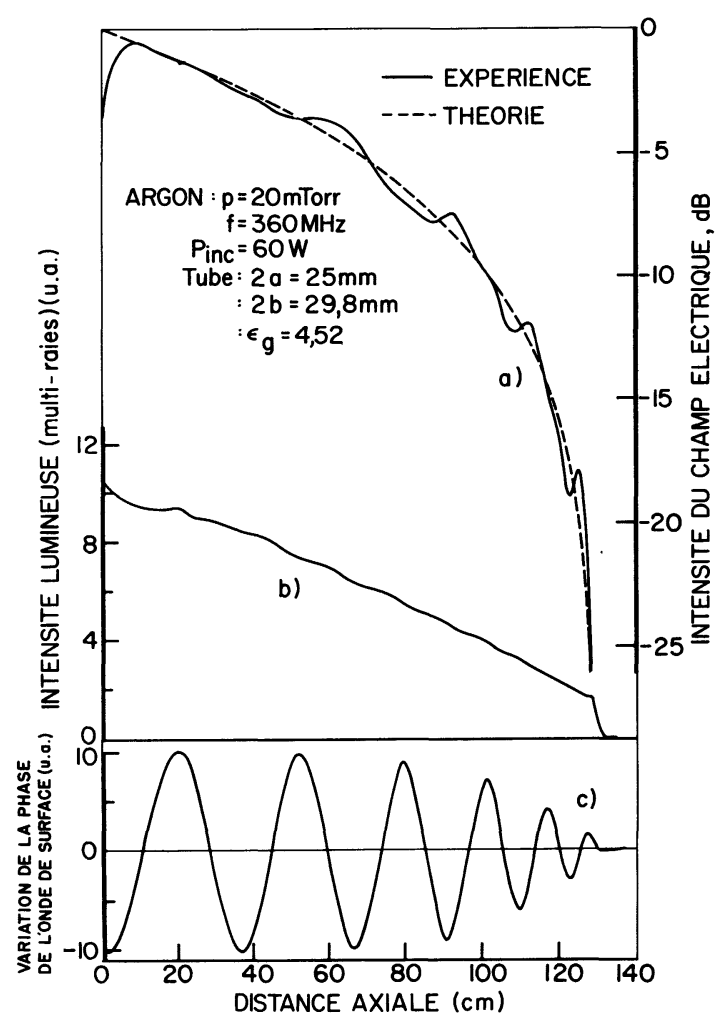

Fig. 2. - $-a$ ) Variation de l'intensité de la composante $E_{\mathrm{r}}$ du champ électrique de l'onde de surface le long de la colonne à partir de l'excitateur. Le plasma est entretenu par l'onde elle-même. $b$ ) Variation correspondante de l'intensité lumineuse totale émise. Elle est proportionnelle à la densité électronique. c) Interférogramme HF montrant la variation de phase de l'onde de surface le long de la colonne de plasma.

[a) Intensity variation of the radial component of the surface wave electric field as a function of position along the plasma column, starting from the wave launcher. The plasma is produced by the surface wave. b) Corresponding variation of total emitted light intensity. It is proportional to the electron density. c) RF interferogram showing the surface wave phase variation along the plasma column.]

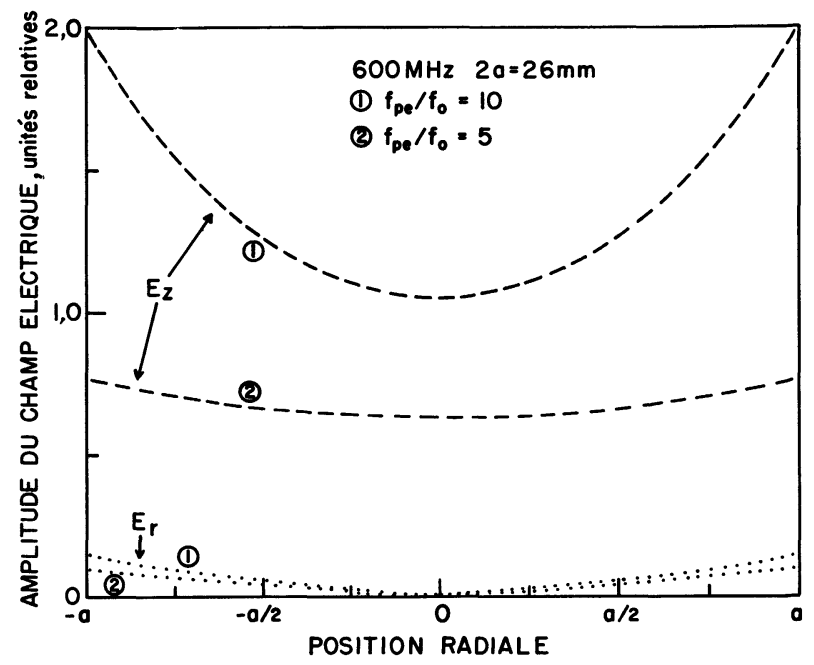

Fig. 3. - Valeur calculée des composantes $E_{z}(r)$ et $E_{\mathrm{r}}(r)$ dans un plasma de rayon $a$, pour deux valeurs du rapport $f_{\text {pe }} / f_{0}$ (modèle du plasma homogène).

[Calculated value of $E_{z}(r)$ and $E_{\mathrm{r}}(r)$ inside a plasma of radius $a$, for two values of $f_{\mathrm{pe}} / f_{0}$ (homogeneous plasma model).]

où $A_{1}$ et $A_{2}$ sont des constantes, $I_{0}, I_{1}$, les fonctions de Bessel modifiées de première espèce et $\beta_{0}=\omega / c$.

$\mathrm{Ce}$ modèle (plasma homogène) permet donc de connaître l'équation de dispersion ainsi que la distribution du champ électromagnétique. Si l'on tient compte de l'inhomogénéité radiale $(n=n(r))$, cette distribution est modifiée (spécialement pour $E_{\mathrm{r}}$ ) et il faudra en tenir compte pour la distribution radiale des particules du plasma.

2.2 CAS D'UN PLASMA INHOMOGÈNE RADIALEMENT. - Dans un plasma de forme cylindrique dont la permittivité varie radialement, pour déterminer la composante $E_{z}(r)$ d'une onde de surface à symétrie azimutale, il faut résoudre l'équation différentielle suivante :

$$
\begin{aligned}
\frac{\mathrm{d}^{2} E_{\mathrm{z}}}{\mathrm{d} r^{2}}+\left\{\frac{1}{r}-\right. & \left.\frac{\mathrm{d} \varepsilon_{\mathrm{p}}(r)}{\mathrm{d} r} \frac{1}{\varepsilon_{\mathrm{p}}(r)} \frac{\beta^{2}}{\beta_{0}^{2} \varepsilon_{\mathrm{p}}(r)-\beta^{2}}\right\} \times \\
& \times \frac{\mathrm{d} E_{z}}{\mathrm{~d} r}+\left(\beta_{0}^{2} \varepsilon_{\mathrm{p}}(r)-\beta^{2}\right) E_{\mathrm{z}}=0 .
\end{aligned}
$$

Quant à la composante $E_{\mathrm{r}}(r)$, elle découle de $E_{z}(r)$ par la relation :

$$
E_{\mathrm{r}}(r)=-\frac{\beta}{\beta_{0}^{2} \varepsilon_{\mathrm{p}}(r)-\beta^{2}} \frac{\mathrm{d} E_{z}}{\mathrm{~d} r}
$$

et $E_{\varphi}$ est nulle pour une onde TM.

A titre d'exemple, nous avons résolu les équations (3) et (4) avec une distribution de Bessel pour la densité électronique :

$$
n(r)=n(0) J_{0}(\mu r / a)
$$


où $\mu$ est une constante égale ou inférieure à 2,4 qui fixe la valeur de la densité sur la paroi et $a$ le rayon du plasma. Nous montrerons en section 4.1 que ce type de distribution est une bonne approximation.

Dans ce cas, la permittivité s'écrit :

$$
\varepsilon_{\mathrm{p}}(r)=1-\frac{\omega_{\mathrm{pe}}^{2}(0)}{\omega^{2}} J_{0}(\mu r / a) .
$$

La résolution de l'équation (3), possible si $\omega_{\mathrm{pe}}(a)>\omega$, et l'équation (4) montrent (Fig. 4) que c'est surtout la composante $E_{\mathrm{r}}(r)$ qui se trouve affectée par l'introduction d'un gradient de densité et ce

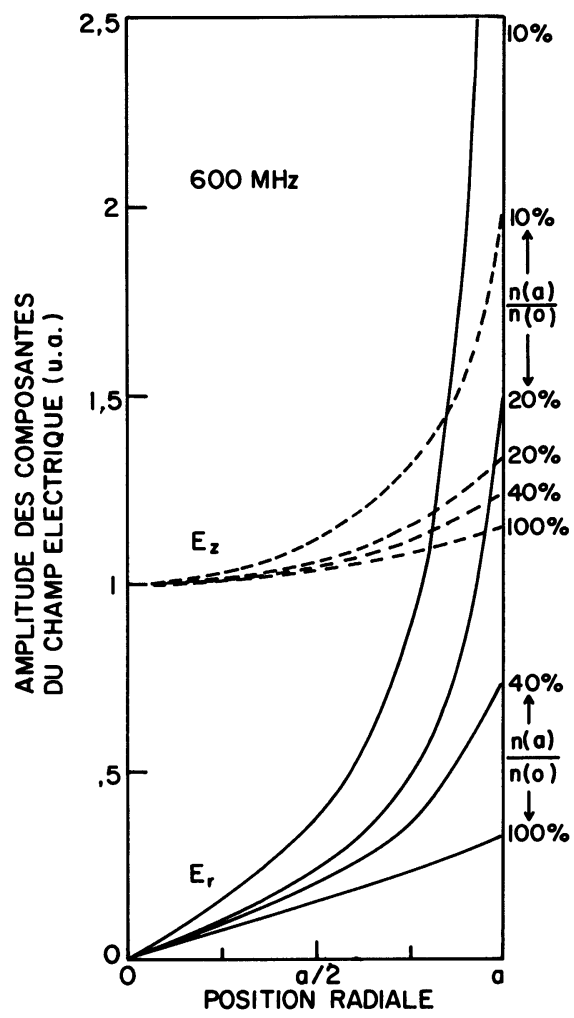

Fig. 4. - Valeur calculée de $E_{z}(r)$ et $E_{\mathrm{r}}(r)$ dans un plasma de densité électronique $n(0) J_{0}(\mu r / a)$ (modèle du plasma inhomogène). Le paramètre est $J_{0}(\mu)=n(a) / n(0)$, la valeur de densité électronique sur la paroi relativement à celle sur l'axe.

[Calculated value of $E_{z}(r)$ and $E_{\mathrm{r}}(r)$ inside a plasma of electron density $n(0) J_{0}(\mu r / a)$ (inhomogeneous plasma model). The parameter is $J_{0}(\mu)=n(a) / n(0)$, the ratio of the electron density at the wall to the central electron density.]

d'autant plus que le rapport $n(a) / n(0)$ est faible. On ne peut donc pas, en général, négliger l'effet de la composante $E_{\mathrm{r}}(r)$ dans le plasma. Dans ce cas, l'action du champ électrique sur les électrons résulte du champ total $E_{\mathrm{T}}(r)$ :

$$
E_{\mathrm{T}}(r)=\sqrt{E_{\mathrm{r}}^{2}(r)+E_{z}^{2}(r)} .
$$

Appelons $E_{\mathrm{Tv}}(r)$, la valeur du champ électrique total obtenue dans le cas d'une distribution électro- nique décrite par la relation (5) pour une valeur donnée de $n(a) / n(0)$, et désignons par $E_{\mathrm{Tc}}(r)$ la valeur obtenue (essentiellement $E_{z}(r)$ ) dans le cas où $n(r)$ est constant. La figure 5 montre que la courbure du champ $E_{\mathrm{Tv}}$ (représenté par les cercles pleins) est plus forte que

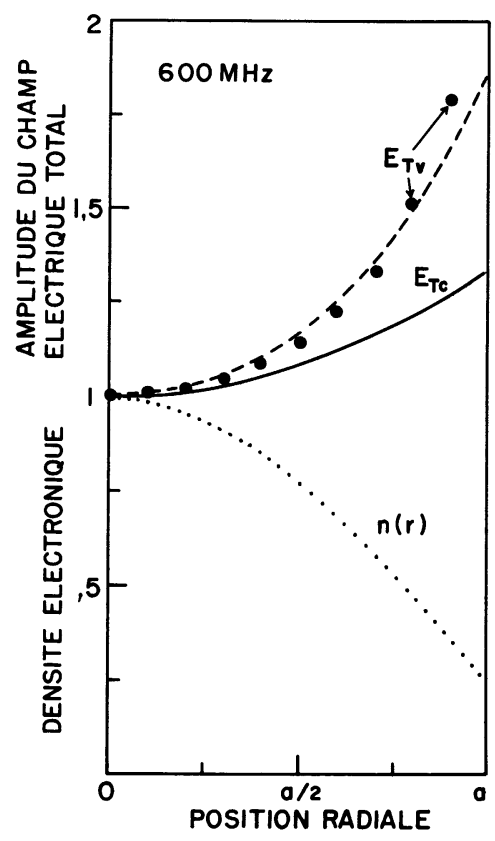

Fig. 5. $-E_{\mathrm{Tc}}$ (trait plein) : champ total de l'onde de surface calculé dans le cas du plasma homogène $\left(f_{\text {pe }} / f_{0}=2,7\right) . E_{\mathrm{Tr}}(\bullet)$ : champ total calculé dans le cas d'un plasma inhomogène en supposant une densité électronique $n(0) J_{0}(\mu r / a)$ (représentée en pointillé) avec $f_{\overline{\mathrm{pe}}} / f_{0}=2,7$ et $\mu=1,95$. Les points $E_{\mathrm{Tv}}$ sont lissés par l'expression $I_{0}(\sigma r)$ (trait discontinu) avec $\sigma=1,29 \mathrm{~cm}^{-1}$ et $a=1,3 \mathrm{~cm}$.

$\left[E_{\mathrm{Tc}}\right.$ (full line) : surface wave total electric field calculated for a homogeneous plasma $\left(f_{\mathrm{pe}} / f_{0}=2.7\right) . E_{\mathrm{Tv}}(\bullet)$ : total electric field calculated for an inhomogeneous plasma. The assumed electron density distribution (dotted line) is given by $n(0) J_{0}(\mu r / a)$ with $f_{\overline{\mathrm{pe}}} / f_{0}=2.7$ and $\mu=1.95$. The $E_{\mathrm{Tv}}$ points are fitted by the expression $I_{0}(\sigma r)$ (dashed line) with $\sigma=1.29 \mathrm{~cm}^{-1}$ and $a=1.3 \mathrm{~cm}$.]

celle de $E_{\mathrm{Tc}}$ donnée par le modèle homogène pour une même densité électronique moyenne $\langle n\rangle$ où

$$
\langle n\rangle=\frac{2}{a^{2}} \int_{0}^{a} n(r) r \mathrm{~d} r .
$$

A cette densité moyenne correspond une fréquence moyenne du plasma,

$$
f_{\overline{\mathrm{pe}}}=\frac{1}{2 \pi}\left(\langle n\rangle e^{2} / m \varepsilon_{0}\right)^{1 / 2} .
$$

(Noter que la valeur des champs $E_{\mathrm{Tv}}$ et $E_{\mathrm{Tc}}$ de la figure 5 est arbitrairement normalisée à l'unité sur l'axe.)

On peut trouver une bonne approximation pour le champ $E_{\mathrm{Tv}}$ (Fig. 5) en utilisant une expression analytique de la forme :

$$
E_{\mathrm{T}}(r)=I_{0}(\sigma r)
$$


où $\sigma$ est un paramètre d'ajustement qui ne peut être calculé analytiquement et qui représente la courbure du champ.

D'autre part, on verrait [7] que la courbe de dispersion théorique obtenue dans le cas du plasma homogène est finalement peu affectée par la prise en compte de l'inhomogénéité radiale si l'on exclut les valeurs de $\beta a \gtrsim 1$ qui ne correspondent pas à notre domaine d'observation.

Un paramètre important pour les calculs du profil des champs électriques dans le plasma inhomogène est la densité électronique moyenne $\langle n\rangle$. Celle-ci peut être déterminée en différents points le long de la colonne d'après la mesure de la décroissance de la composante $E_{\mathrm{r}}(r)$ à partir de la paroi de verre à l'extérieur du tube [7]. Les résultats ainsi obtenus (Fig. 6) sont comparables avec ceux fournis par une cavité résonnante $\mathrm{TM}_{010}$ [2].

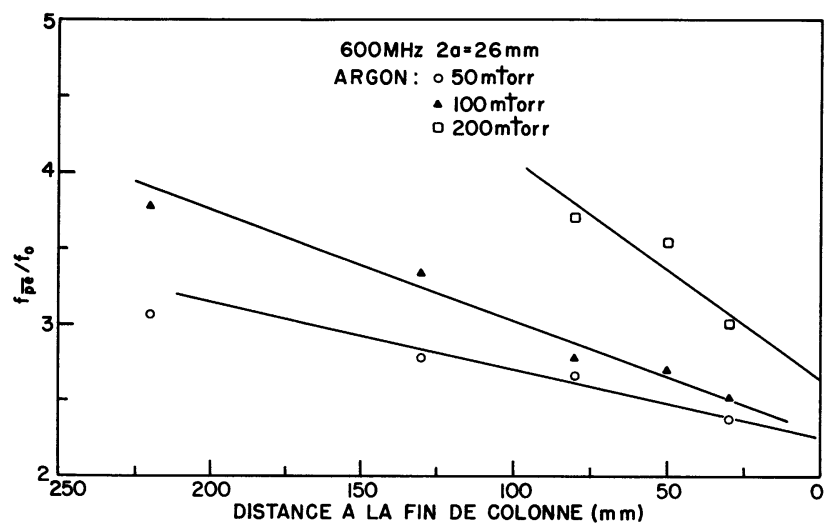

Fig. 6. - Valeur mesurée de $f_{\overline{\mathrm{pe}}} / f_{0}$ le long de la colonne déterminée à partir de la décroissance dans l'air de la composante $E_{\mathrm{r}}(r)$.

[Measured $f_{\overline{\mathrm{pe}}} / f_{0}$ value along the plasma column from the $E_{\mathrm{r}}(r)$ radial decrease away from the column in air.]

3. Résultats expérimentaux. - 3.1 DisPoSITIF EXPÉRIMENTAL. - La figure 7 représente de façon schématique le montage expérimental utilisé. Le circuit de mesure et d'alimentation HF n'est pas représenté. On trouvera dans [3] toutes les informations concernant le surfatron. Dans le cas présent, on peut, selon les besoins, produire le plasma à 300,600 ou $1000 \mathrm{MHz}$, avec une puissance HF incidente toujours totalement absorbée.

On sait que la longueur d'une colonne de plasma d'onde de surface est une fonction croissante de la puissance HF absorbée [1]. Dans ce qui suit, on s'est limité à des puissances telles que le plasma ne touche pas vraiment l'une ou l'autre des extrémités du tube. On évite ainsi la réflexion de l'onde de surface i.e. la formation d'ondes stationnaires. Par ailleurs, la longueur du plasma a été choisie de sorte que les coefficients d'absorption ne soient pas trop élevés. On a ainsi toujours utilisé des tubes en pyrex $\left(\varepsilon_{\mathrm{g}}=4,52\right)$, de $26 \mathrm{~mm}$ de diamètre intérieur et de $200 \mathrm{~mm}$ de

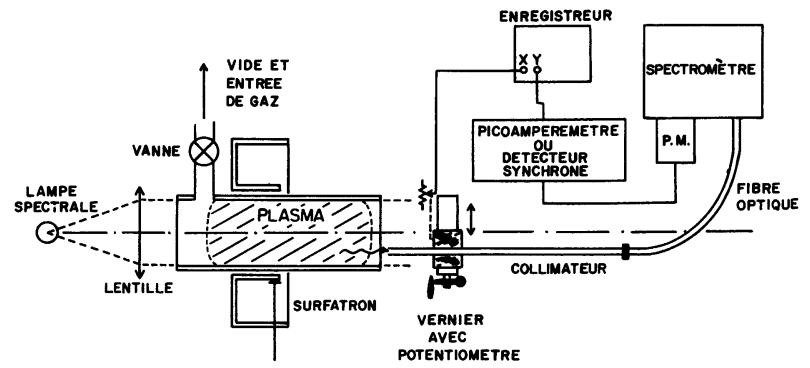

Fig. 7. - Dispositif expérimental.

[Experimental arrangement.]

longueur. Chaque tube se trouve fermé aux deux extrémités par des fenêtres (pyrex) disposées perpendiculairement à son axe et collées avec une résine à faible tension de vapeur (Torr seal). Un bâti à vide assure un vide ultime de l'ordre de $5 \times 10^{-7}$ torr $\left(\simeq 10^{-4} \mathrm{~Pa}\right)$. Une vanne permet d'isoler le tube qui est rempli, avant chaque mesure, à la pression requise. On utilise de l'argon pur à 99,998\% .

La lumière émise par le plasma est examinée à l'aide d'un collimateur placé axialement face à une fenêtre (Fig. 7). Sa longueur $(\simeq 250 \mathrm{~mm})$ est supérieure à celle du plasma. Pour éviter la diffusion de lumière le long de la paroi interne du collimateur, on a disposé un fil très fin enroulé de façon hélicoïdale. Le collimateur permet de recueillir la lumière du plasma se trouvant dans un cylindre axial d'environ $1 \mathrm{~mm}$ de diamètre. Le déplacement radial du collimateur est assuré par un mécanisme à vernier qui est relié à un potentiomètre pour l'enregistrement de la position. La lumière ainsi collimatée est transmise par une fibre optique à un spectromètre à réseau (Jarrel-Ash de $0,25 \mathrm{~m}$ ) dont la fente de sortie éclaire un photomultiplicateur Hamamatsu de type R-636, relié à un picoampèremètre.

Le montage peut également servir à des mesures par absorption optique. Il suffit pour cela d'installer face à l'autre fenêtre une lampe spectrale (vg Philips) suivie d'une lentille pour former un faisceau parallèle ; on vise en général la lueur négative qui est modulée par le secteur à $60 \mathrm{~Hz}$. On utilise un détecteur synchrone sur cette fréquence pour distinguer, dans l'intensité totale reçue sur le photomultiplicateur, la contribution provenant de la lampe spectrale. On connaît les caractéristiques des raies de cette lampe grâce à un étalonnage avec un interféromètre FabryPérot réalisé au laboratoire de Physique des Plasmas d'Orsay.

La sortie du picoampèremètre ou du détecteur synchrone est reliée à la borne $Y$ d'un traceur $X-Y$ et le potentiomètre de position du collimateur à la borne $X$; il suffit de déplacer le collimateur pour obtenir la variation radiale de l'intensité lumineuse de la raie choisie. La répétitivité des enregistrements obtenus est très bonne.

Ce montage permet aussi d'étudier de la même façon la distribution radiale des atomes excités d'un 
plasma de colonne positive. Celui-ci est produit dans un tube de mêmes dimensions, auquel on a ajouté des bras latéraux pour les électrodes [4]. Celles-ci ont été dégazées par bombardement électronique; on a utilisé une cathode chaude à oxydes (strontium, baryum et rubidium).

3.2 Distribution Radiale Des atomes Radiatifs DE L'ARGON. - Nous avons examiné la distribution radiale d'un certain nombre de transitions optiques de l'argon, dont les niveaux sont représentés sur la figure 8. Quelques raies spectrales caractéristiques provenant des niveaux $3 p^{5} 4 s$ à $8 s, 3 p^{5} 4 p$ à $6 p$ et $3 \mathrm{p}^{5} 4 \mathrm{~d}$ à $10 \mathrm{~d}$ y sont indiquées.

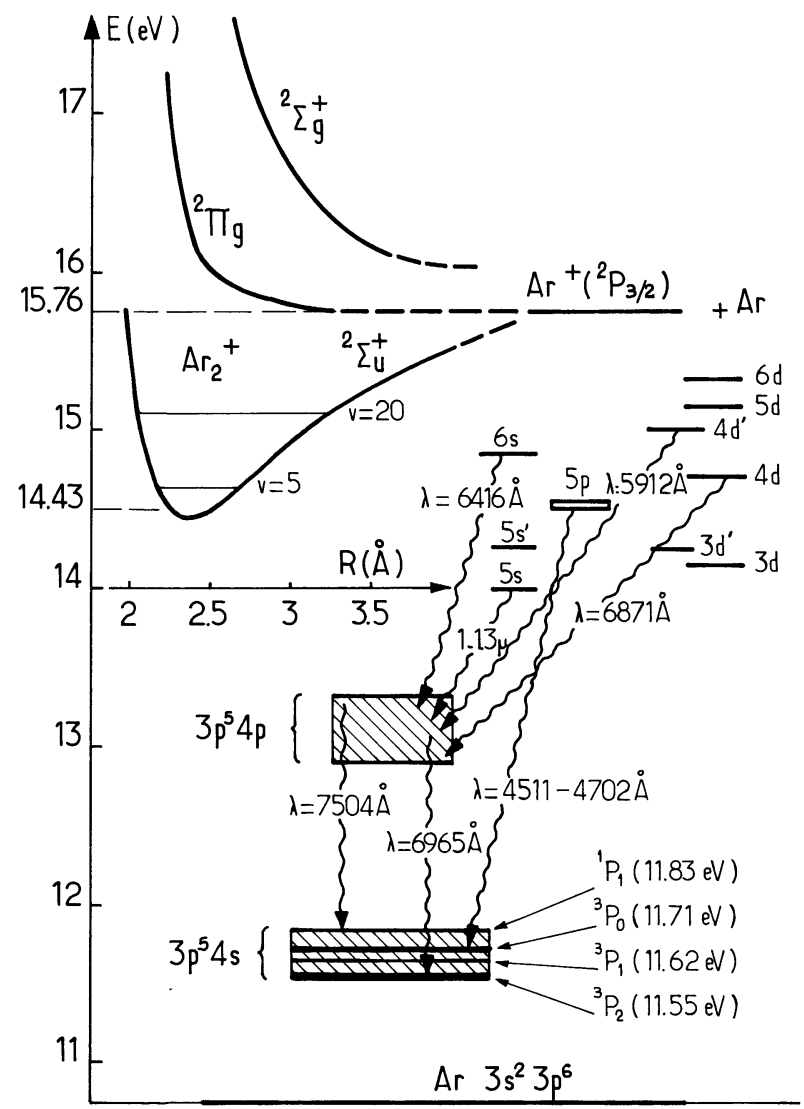

Fig. 8. - Niveaux d'énergie des atomes et ions excités dans l'argon.

[Energy levels of argon excited atoms and ions.]

Nous avons pris soin de choisir une transition à force d'oscillateur faible et dont le niveau inférieur est peu peuplé comme par exemple la raie $549,6 \mathrm{~nm}$ de la transition $3 p^{5} 6 \mathrm{~d} \rightarrow 4 \mathrm{p}$ (force d'oscillateur $\simeq 10^{-2}$ ).

La distribution radiale de l'intensité de la raie $549,6 \mathrm{~nm}$ a été enregistrée pour différentes pressions d'argon comprises entre 50 et 200 mtorr $(6,5$ à $26 \mathrm{~Pa})$. La puissance HF est toujours fixée de sorte que le plasma remplisse le tube d'expérience sans se rendre jusqu'aux fenêtres de sortie de façon à éviter les réflexions d'ondes. On maintient ainsi une onde progressive qui correspond au mode de propagation que nous connaissons théoriquement et expérimentalement [2].

Pratiquement, la colonne de plasma étudiée n'excède pas $200 \mathrm{~mm}$ de long $(100 \mathrm{~mm}$ de part et d'autre du point d'excitation de l'onde de surface). Les figures 2 et 6 montrent qu'il existe un gradient de densité longitudinal. L'amplitude de variation de $n$ est toutefois limitée par l'existence d'une densité minimum critique en dessous de laquelle l'onde de surface ne peut se propager. La figure 6 montre que pour une colonne de $100 \mathrm{~mm} n$ varie d'environ un facteur deux. Le profil radial que l'on observe est donc un profil moyen, que l'on peut attribuer à une densité moyenne. Ce profil radial est toutefois peu sensible à la longueur du plasma comme le montre la figure 9 où l'on a enregistré l'émission de la raie $549,6 \mathrm{~nm}$ pour les deux puissances 0,7 et $1,8 \mathrm{~W}$. A ces puissances, la longueur de la colonne de plasma est respectivement de 100 et $200 \mathrm{~mm}$.

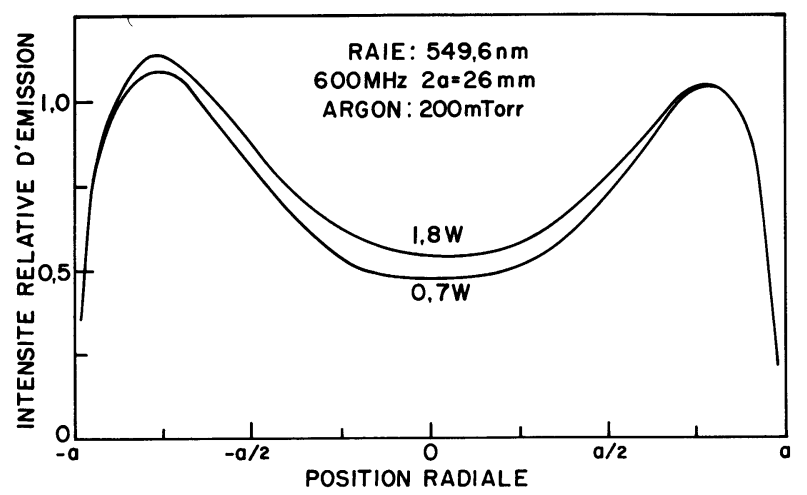

Fig. 9. - Distribution radiale de l'intensité relative d'une raie optiquement mince pour deux puissances $\mathrm{HF}:(0,7 \mathrm{~W}$, longueur de plasma $\left.L_{\mathrm{p}}=10 \mathrm{~cm} ; 1,8 \mathrm{~W}, L_{\mathrm{p}}=20 \mathrm{~cm}\right)$.

[Measured radial distribution of the relative intensity emitted by an optically thin line, for two $\mathrm{HF}$ powers : $(0.7 \mathrm{~W}$, plasma length $\left.L_{\mathrm{p}}=10 \mathrm{~cm} ; 1.8 \mathrm{~W}, L_{\mathrm{p}}=20 \mathrm{~cm}\right)$.]

Les résultats obtenus à la fréquence de $600 \mathrm{MHz}$ sont représentés sur la figure 10 , la puissance HF absorbée étant de l'ordre de $2 \mathrm{~W}$. On constate que la

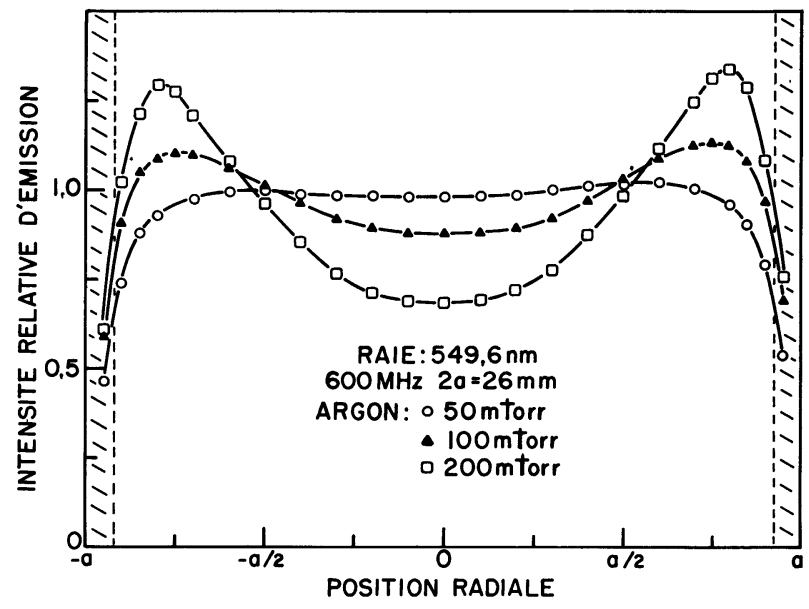

Fig. 10. - Distribution radiale de l'intensité relative d'une raie optiquement mince mesurée, pour trois pressions de gaz, avec $f_{0}=600 \mathrm{MHz}$.

[Measured radial distribution of the relative intensity emitted by an optically thin line. for three gas pressures. with $f_{0}=600 \mathrm{M} \mathrm{Hz}$.] 
distribution est plate à 50 mtorr $(6,5 \mathrm{~Pa})$ et qu'elle se creuse ensuite, d'autant plus que la pression augmente. On remarque aussi que les maximums d'intensité qui sont apparus, se rapprochent des parois avec l'accroissement de pression. Les intensités des différents enregistrements ont été normalisées, de façon à ce que les extremums des distributions soient symétriques par rapport à la valeur un. Notons enfin qu'au voisinage immédiat des bords du tube à décharge, les intensités relevées sont faussées à cause de la résolution finie $(\simeq 1 \mathrm{~mm})$ du collimateur. On ne tiendra pas compte de cette région (zone hachurée) dans l'interprétation théorique (Sect. 5).

L'influence de la fréquence de l'onde de surface sur la distribution radiale de l'émission apparaît sur les figures 11 et 12 . Comparativement à $600 \mathrm{MHz}$

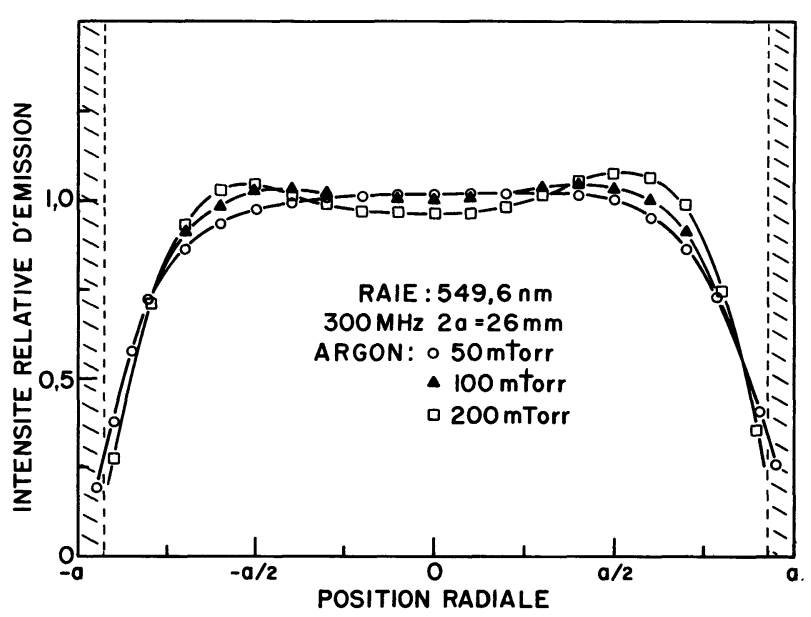

Fig. 11. - Distribution radiale de l'intensité relative d'une raie optiquement mince mesurée, pour trois pressions de gaz, avec $f_{0}=300 \mathrm{MHz}$

[Measured radial distribution of the relative intensity emitted by an optically thin line. for three gas pressures. with $f_{0}=300 \mathrm{MHz}$.]

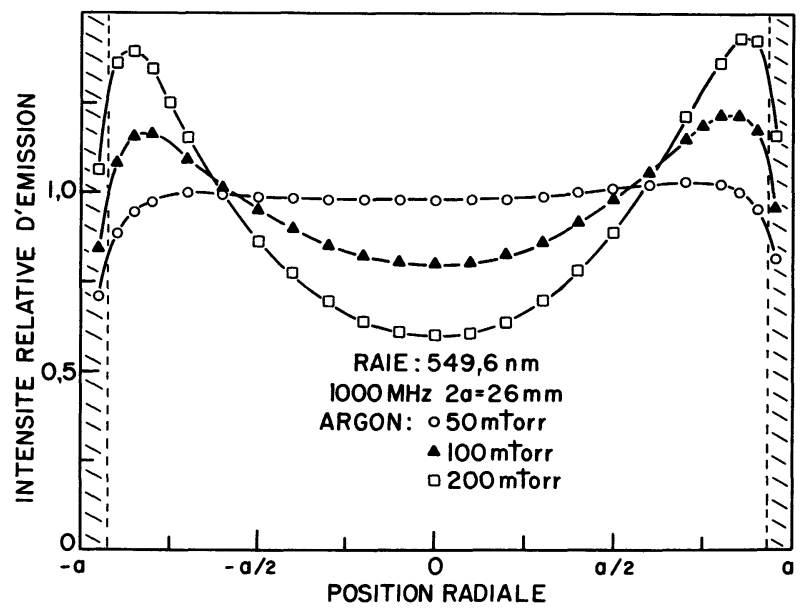

Fig. 12. - Distribution radiale de l'intensité relative d'une raie optiquement mince mesurée, pour trois pressions de gaz, avec $f_{0}=1000 \mathrm{MHz}$

[Measured radial distribution of the relative intensity emitted by an optically thin line. for three gas pressures, with $f_{0}=1000 \mathrm{MHz}$.]
(Fig. 10), on constate qu'à $300 \mathrm{MHz}$, les distributions sont à peu près plates pour les trois pressions de référence, alors qu'elles sont plus creuses sur l'axe à $1000 \mathrm{MHz}$, sauf pour 50 mtorr. On notera qu'à 50 mtorr, la distribution radiale présente un plateau pour les trois fréquences étudiées et que la largeur de ce plateau augmente avec la fréquence. On remarquera également que, relativement à sa valeur sur l'axe, l'intensité lumineuse au voisinage immédiat de la paroi augmente avec la fréquence.

Des résultats semblables ont été obtenus avec une autre raie optiquement mince (Arl 591,2 $\mathrm{nm}$, voir Fig. 8).

3.3 Distribution RADiale Des atomes mÉtastaBLES DE L'ARGON. - On a déterminé la densité des atomes métastables de l'argon par absorption optique suivant le montage de la figure 7. Une lampe spectrale d'argon, placée à une extrémité du tube à décharge, envoie un faisceau de lumière parallèle. A l'autre extrémité, le collimateur, conjugué avec une détection synchrone, recueille la lumière de la lampe transmise à travers le plasma en fonction de la position radiale. En comparant avec le même enregistrement sans plasma, on obtient le coefficient d'absorption de la raie, $A_{\mathrm{L}}(r)$, dont on peut déduire la densité $n_{\mathrm{M}}(r)$ [8]. Dans le cas où $A_{\mathrm{L}}(r)<0,1$, la densité de population $n_{\mathrm{M}}(r)$ est simplement proportionnelle à l'absorption.

Nous avons procédé ainsi pour obtenir le coefficient d'absorption de la raie $696,5 \mathrm{~nm}$ qui conduit à la densité de population des métastables $\operatorname{Ar}\left({ }^{3} \mathrm{P}_{2}\right)$. La figure 13 présente la distribution 'radiale de densité des métastables $\operatorname{Ar}\left({ }^{3} \mathrm{P}_{2}\right)$ dans de l'argon pur. On constate que comparativement à la distribution de densité des atomes radiatifs, la distribution des métastables se creuse beaucoup moins vite avec la pression. En effet, même à 350 mtorr, la distribution

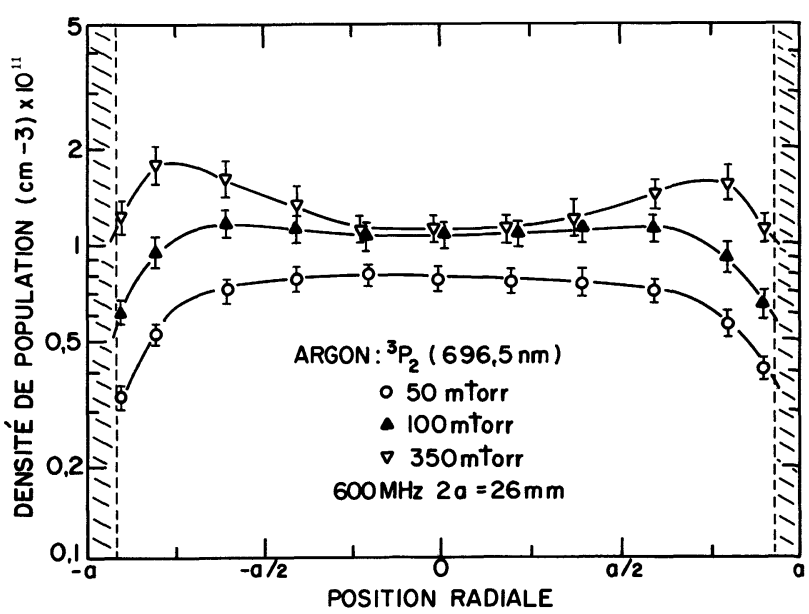

Fig. 13. - Distribution radiale du niveau métastable ${ }^{3} \mathbf{P}_{2}$ dans un plasma d'argon pur produit par l'onde de surface, en l'absence de réflexions sur les extrémités du tube.

[Radial population-density distribution of the ${ }^{3} \mathrm{P}_{2}$ metastable atoms in a pure argon surface wave produced plasma, in the absence of reflections on the column ends.] 
des métastables est encore moins creuse que celle des atomes radiatifs a $100 \mathrm{mtorr}$ (Fig 10).

3. 4 DisTRIBUTION DES ATOMES EXCITÉS DANS DES MÉI.ANGES ARGON-XÉNON ET ARGON-AZOTE. - Nous utilisons maintenant des mélanges $90 \%$ argon- $10 \%$ xénon et $90 \%$ argon- $10 \%$ azote pour étudier la distribution radiale des atomes métastables de l'argon dans une situation où les termes de perte par diffusion et réexcitation électronique sont négligeables devant ceux dus aux collisions avec les atomes neutres du xénon ou avec les molécules d'azote (voir 4.2.2 et 5.2).

Nous avons procédé comme en 3.3 pour obtenir le coefficient d'absorption de la raie $696,5 \mathrm{~nm}$ qui permet de déterminer la densité de population des métastables $\operatorname{Ar}\left({ }^{3} \mathrm{P}_{2}\right)$. Cependant, la concentration des atomes métastables étant fortement réduite par la présence des neutres de l'azote ou du xénon, les valeurs du coefficient d'absorption observées sont maintenant très faibles $(<5 \%)$ et de ce fait affectées par les fluctuations de la lampe. Ceci explique les larges barres d'erreur qui entourent les valeurs d'absorption sur la figure 14. Ce profil d'absorption montre un creux central beaucoup plus marqué que dans le cas de l'argon pur (Fig. 13) où la diffusion et la destruction par les électrons ne sont pas négligeables. On note, d'autre part, que le profil d'émission de la raie $696,5 \mathrm{~nm}$, aplati par autoabsorption dans l'argon pur, présente un creux presqu'aussi profond que celui de la raie optiquement mince $549,6 \mathrm{~nm}$. De fait, ces deux profils d'émission se rejoignent presque complètement

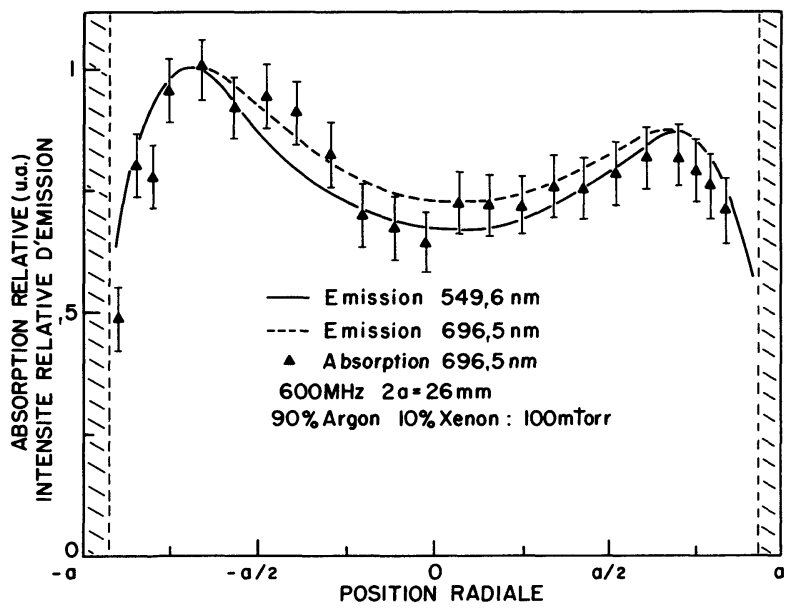

Fig. 14. - Comparaison dans un mélange argon-xénon de la variation radiale de l'émission d'une raie optiquement mince $(549,6 \mathrm{~nm})$ avec celle d'une raie qui était autoabsorbée dans l'argon pur $(696,5 \mathrm{~nm})$. Egalement, variation radiale du coefficient d'absorption de la raie $696,5 \mathrm{~nm}$ (niveau métastable $\operatorname{Ar}{ }^{3} \mathrm{P}_{2}$ ); la valeur maximum correspond à une absorption de 0.04 .

[Comparison of the radial variation of emission in a $100 \mathrm{mtorr}$ argon-xenon mixture of a line $(696.5 \mathrm{~nm})$ that was self-absorbed in pure argon with an optically thin line $(549.6 \mathrm{~nm})$. Also shown is the absorption coefficient of the $696.5 \mathrm{~nm}$ line as a function of radius (the maximum value corresponds to an absorption of 0.04 ).] à la pression de 200 mtorr, comme le montre la figure 15. Ceci signifie que les métastables de l'argon sont suffisamment détruits par le xénon pour rendre la raie ArI $696,5 \mathrm{~nm}$ optiquement mince. Enfin, on constate sur la figure 14 que les deux profils d'émission et celui d'absorption, normalisés à un au maximum, présentent des formes très semblables.

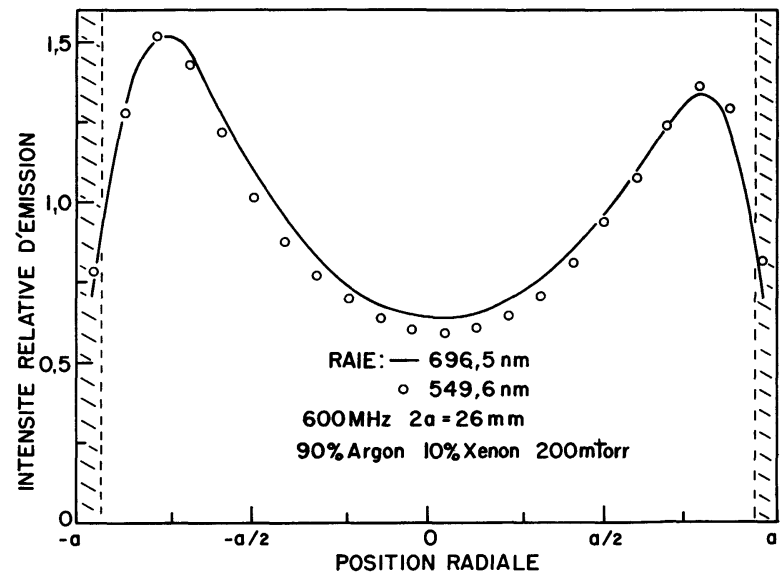

Fig. 15. - Distribution radiale de l'intensité relative d'émission enregistrée dans un mélange argon-xénon, pour une raie optiquement mince $(549,6 \mathrm{~nm})$ et une raie qui était autoabsorbée dans l'argon pur $(696,5 \mathrm{~nm})$.

[Comparison of the radial variation of emission in a $200 \mathrm{mtorr}$ argon-xenon mixture of a line $(696.5 \mathrm{~nm})$ that was self-absorbed in pure argon with an optically thin line $(549.6 \mathrm{~nm})$.]

Une étude semblable a été réalisée dans un mélange $90 \%$ argon- $10 \%$ azote. La figure 16 montre que, comme dans le cas du mélange Ar-Xe, le profil d'émission de la raie $696,5 \mathrm{~nm}$ est pratiquement aussi creusé que celui de la $549,6 \mathrm{~nm}$. De même, le profil d'absorption de la raie $696,5 \mathrm{~nm}$ suit de près les deux profils d'émission. On constate sur les figures 14,

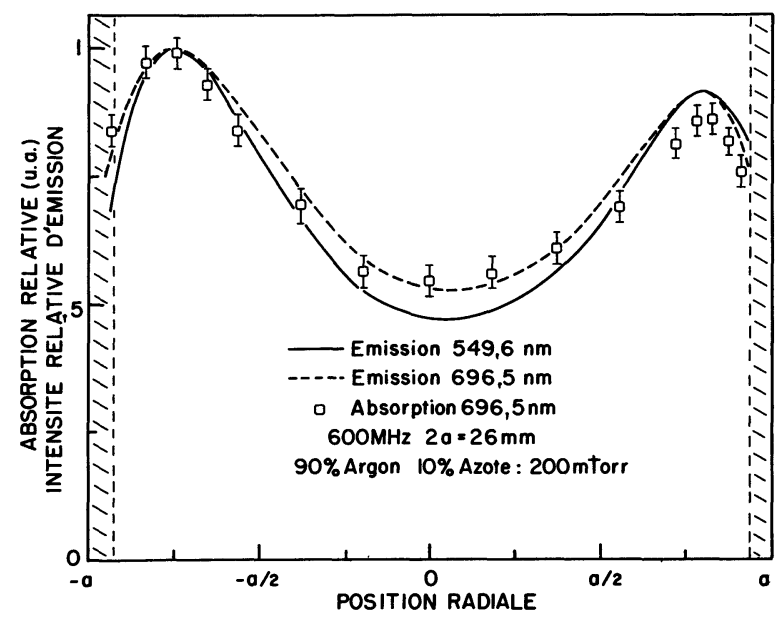

Fig. 16. - Même étude que pour la figure 15 mais dans un mélange argon-azote.

[As for figure 15 with an argon-nitrogen mixture.] 
15 et 16 que les profils d'émission et d'absorption présentent une certaine dissymétrie. Cette dissymétrie provient de la difficulté à centrer l'excitateur suivant l'axe du tube. Les études des mélanges $\mathrm{Ar}-\mathrm{N}_{2}$ et $\mathrm{Ar}-\mathrm{Xe}$ ont été effectuées avec un excitateur de diamètre légèrement supérieur au tube. Dans ce cas, les parois métalliques affectent la propagation de l'onde. Dans le cas de l'argon pur, l'excitateur utilisé était de grand diamètre par rapport au tube et les effets de centrage étaient peu sensibles (cf. Figs. 10, 11 et 12).

4. Calcul de la distribution radiale de densité des électrons et des atomes excités. - Dans cette étude, la pression du gaz est suffisamment faible et le taux d'ionisation si peu élevé que l'on peut affirmer que, dans l'argon pur, les atomes sont principalement excités par collisions électroniques. En conséquence, la répartition radiale des atomes excités se trouve liée à celle des électrons.

Dans les calculs qui suivent, nous supposerons que le corps de la fonction de distribution des vitesses électroniques, en dessous du premier potentiel d'excitation, est maxwellien. La température $T_{\mathrm{e}}$ qui caractérise ce corps demeure constante en fonction $\mathrm{du}$ rayon, malgré la variation radiale du champ électrique HF $\left(^{1}\right)$. Cette remarque est importante pour le calcul de la diffusion des électrons. Par contre, la variation radiale du champ électrique perturbe la fonction de distribution des électrons au-dessus du premier potentiel d'excitation $\left(V_{1}=11,5 \mathrm{~V}\right.$ pour l'argon). On va calculer, en écrivant l'équation de bilan en énergie (gain en énergie par le champ $E$ compensé par les pertes inélastiques) la dépendance des taux de collisions inélastiques $\left\langle\sigma_{\mathrm{j}} w_{\mathrm{e}}\right\rangle\left(\sigma_{\mathrm{j}}\right.$ section efficace d'excitation du niveau $\mathrm{j}, w_{\mathrm{e}}$ vitesse des électrons) avec l'intensité du champ électrique.

4.1 Distribution RADIALE DE LA DENSITÉ ÉLECTRONIQUE. - Les pressions d'argon étudiées sont telles que le libre parcours-moyen pour une collision électron-neutre est bien inférieur au diamètre du plasma; concrètement, les valeurs du produit $p a$ ( $p$ est la pression des neutres en torr et $a$, le rayon du tube exprimé en centimètre) sont comprises entre $2 \times 10^{-2}$ et $2 \mathrm{~cm}$ torr. D'autre part, les valeurs de densités électroniques sont situées approximativement entre $10^{9}$ et $10^{12}$ électrons $/ \mathrm{cm}^{3}$. La diffusion des électrons vers les parois se fait donc de façon ambipolaire. Etant donné, d'autre part, que manifestement $T_{\mathrm{e}} \gg T_{\mathrm{i}}$, où $T_{\mathrm{i}}$ est la température des ions, le coefficient de diffusion ambipolaire $D_{\mathrm{a}}$ est donné par :

$$
D_{\mathrm{a}} \simeq \frac{\kappa T_{\mathrm{e}}}{e} \frac{\mu_{+0}}{p_{0}}
$$

(1) La température électronique moyenne du plasma d'onde de surface a été estimée par l'étude de l'atténuation de l'onde de surface. Les valeurs de $T_{\mathrm{e}}$ trouvées correspondent assez bien au modèle de colonne positive en régime de diffusion [2]. où $\kappa$ est la constante de Boltzmann, $\mu_{+0}$ la mobilité ionique réduite à la pression atmosphérique et $p_{0}$, la pression réduite correspondante. Admettre que $T_{\mathrm{e}}$ ne varie pas avec le rayon entraîne donc que le coefficient de diffusion est indépendant du rayon.

Dans ces conditions, l'équation d'évolution des électrons peut s'écrire :

$$
\frac{\partial n(r)}{\partial t}=v_{\mathrm{i}}(r) n(r)+D_{\mathrm{a}} \nabla^{2} n(r)-\alpha n^{2}(r)
$$

où $v_{\mathrm{i}}$ est la fréquence d'ionisation, de telle sorte que $v_{\mathrm{i}} n$ représente le nombre de particules produites par unité de volume et de temps. Cette quantité va dépendre du champ électrique HF. Quant au terme $\alpha n^{2}(r)$ où $\alpha$ est positif, il représente la perte d'électrons par recombinaison électrons-ions en volume; $\alpha$ est le coefficient de recombinaison en volume.

Toutefois, dans le domaine des produits pa utilisés dans notre expérience, la température électronique $T_{\mathrm{e}}$ est trop forte pour que le terme de recombinaison soit important [9], nous le négligerons.

Les électrons entraînés par le champ électrique HF sont soumis à un mouvement de dérive du fait des collisions. En conséquence, l'énergie gagnée par un électron dans le champ $E$ est donnée par :

$$
\frac{\mathrm{d} W}{\mathrm{~d} t}=e E v_{\mathrm{e}}
$$

où $v_{\mathrm{e}}$ est la vitesse (moyenne) avec laquelle un électron soumis à des collisions progresse dans la direction du champ. Cette vitesse de dérive est liée au champ par l'intermédiaire de la mobilité électronique ; compte tenu de l'expression de cette dernière, il vient :

$$
\frac{\mathrm{d} W}{\mathrm{~d} t}=\frac{e^{2} E^{2}}{m v}\left\{\frac{v^{2}}{\omega^{2}+v^{2}}\right\}
$$

Notons que $v$, la fréquence de collisions électronneutre pour le transfert de la quantité de mouvement ne dépend que de la température $T_{\mathrm{e}}$ du corps de la fonction de distribution des électrons. Elle est donc indépendante de la position radiale.

L'énergie acquise par l'électron dans le champ HF passe finalement en collisions élastiques et inélastiques et l'on peut écrire :

$$
\begin{array}{r}
\left\{\frac{e^{2}}{m}\left(\frac{v}{\omega^{2}+v^{2}}\right)\right\} E^{2}(r) \simeq v_{\mathrm{i}} e V_{\mathrm{i}}+\sum_{\mathrm{j}} v_{\mathrm{j}} e V_{\mathrm{j}}+ \\
+3(m / M) v \kappa T_{\mathrm{e}} / e
\end{array}
$$

où $v_{\mathrm{j}}$ est la fréquence des collisions conduisant au niveau $\mathrm{j}$, de potentiel $V_{\mathrm{j}} ; V_{\mathrm{i}}$ est le potentiel d'ionisation de l'atome et $M$, la masse de l'ion. L'équation (14) suppose des collisions inélastiques sur des atomes dans l'état fondamental et néglige donc d'éventuelles excitations ou ionisations par étapes. Les mécanismes en plusieurs étapes pourraient être importants dans le bilan d'énergie si les niveaux métastables étaient 
fortement peuplés. Dans ce cas, il conviendrait d'écrire les termes inélastiques dans (14) suivant l'équation :

$$
v_{\mathrm{j}} e V_{\mathrm{j}}=n_{0} C_{0 \mathrm{j}} e V_{0 \mathrm{j}}+n_{\mathrm{M}} C_{\mathrm{Mj}} e V_{\mathrm{Mj}}
$$

où les indices $0, M$ et $\mathrm{j}$ désignent respectivement, le niveau fondamental, le niveau métastable et le niveau radiatif; une paire d'indices indique le niveau initial et final de la transition. Les coefficients $C_{0 \mathbf{j}}$ et $C_{\mathbf{M j}}$ représentent les produits section efficace-vitesse des électrons, moyennés sur la fonction de distribution des vitesses électroniques. On peut estimer l'ordre de grandeur des termes de l'équation (15) en prenant les valeurs numériques suivantes, adaptées aux niveaux $4 p$ (voir Fig. 8) :

$$
\begin{gathered}
C_{0 \mathrm{j}} \simeq 10^{-11} \mathrm{~cm}^{3} \mathrm{~s}^{-1}, \quad C_{\mathrm{Mj}} \simeq 10^{-7} \mathrm{~cm}^{3} \mathrm{~s}^{-1}, \\
V_{0 \mathrm{j}}=13 \mathrm{eV}, \quad V_{\mathrm{Mj}}=1,5 \mathrm{eV}[10] .
\end{gathered}
$$

On trouve ainsi que l'apport des métastables commence a être négligeable à partir de $n_{\mathrm{M}} / n_{0}<9 \times 10^{-4}$. Les résultats expérimentaux (Fig. 13) montrent qu'en fait $n_{\mathrm{M}} / n_{0}$ est compris entre $10^{-4}$ et $10^{-5}$. On peut donc négliger les collisions sur les métastables dans le bilan d'énergie représenté par l'équation (14).

Le terme $3(m / M) v \kappa T_{\mathrm{e}} / e$ représente les pertes d'énergie dues aux collisions élastiques. Aux très faibles valeurs de champ, $T$ augmente comme $E^{2}$ mais aussitôt que les collisions inélastiques dominent les pertes, ce sont ces collisions inélastiques qui vont dépendre de $E^{2}$ alors que $T_{\mathrm{e}}$ sera à peu près constant (la température $T_{\mathrm{e}}$ se stabilise en général à $1 / 3$ ou $1 / 4$ du premier potentiel d'excitation). Nous ferons l'hypothèse que la fréquence d'ionisation $v_{i}$ et les fréquences d'excitation $v_{\mathrm{j}}$ varient comme $E^{2}$ et nous écrirons :

$$
\begin{aligned}
& v_{\mathrm{i}}=A_{\mathrm{i}} E^{2}(r) \\
& v_{\mathrm{j}}=A_{\mathrm{j}} E^{2}(r)
\end{aligned}
$$

où $A_{\mathrm{i}}$ et $A_{\mathrm{j}}$ sont des constantes qui ne dépendent pas de $r$.

A l'état stationnaire pour un plasma de forme cylindrique où les pertes ont lieu exclusivement par diffusion, l'équation (11) s'écrit, compte tenu de $v_{\mathrm{i}}(16)$ et de $E(r)(9)$ :

$$
\frac{\mathrm{d}^{2} n}{\mathrm{~d} r^{2}}+\frac{1}{r} \frac{\mathrm{d} n}{\mathrm{~d} r}+\frac{A_{\mathrm{i}}}{D_{\mathrm{a}}} I_{0}^{2}(\sigma r) n=0 .
$$

On a négligé le terme $\mathrm{d}^{2} n / \mathrm{d} z^{2}$ en considérant que la longueur axiale du plasma serait plusieurs fois plus grande que son diamètre. Pour ce qui est des conditions aux limites nécessaires à la solution de cette équation, on prend $(\mathrm{d} n / \mathrm{d} r)=0$ sur l'axe mais on ne peut $a$ priori imposer $n(a)=0$ car, par exemple, il peut exister un certain pourcentage de particules réfléchies par les parois, ce qui signifie que la recombinaison sur la paroi n'est pas totale. On tiendra compte des résultats expérimentaux pour la valeur à donner à $n(a)$. Cette valeur est importante car elle détermine le terme $A_{\mathrm{i}} / D_{\mathrm{a}}$.

L'équation différentielle a été résolue numériquement sur ordinateur par la méthode Runge-Kutta à l'aide d'un programme IMSL. La figure 17 nous en donne un exemple dans le cas d'un rapport de champ $E_{\mathrm{T}}(a) / E_{\mathrm{T}}(0)=I_{0}(\sigma a)=1,9$. La densité électronique $n(r)$, représentée par des points, est normalisée à la valeur un sur l'axe et on a pris $n(a)=0,2$. Sur la même figure et pour la même valeur $n(a)$, apparaît en point-trait la solution $n(r)$ pour un champ $E$ constant radialement (une telle solution est représentée par une fonction de Bessel $J_{0}$ ). On constate que le profil électronique en présence de champ HF est simplement légèrement plus plat que celui décrit par la fonction de Bessel $J_{0}$.

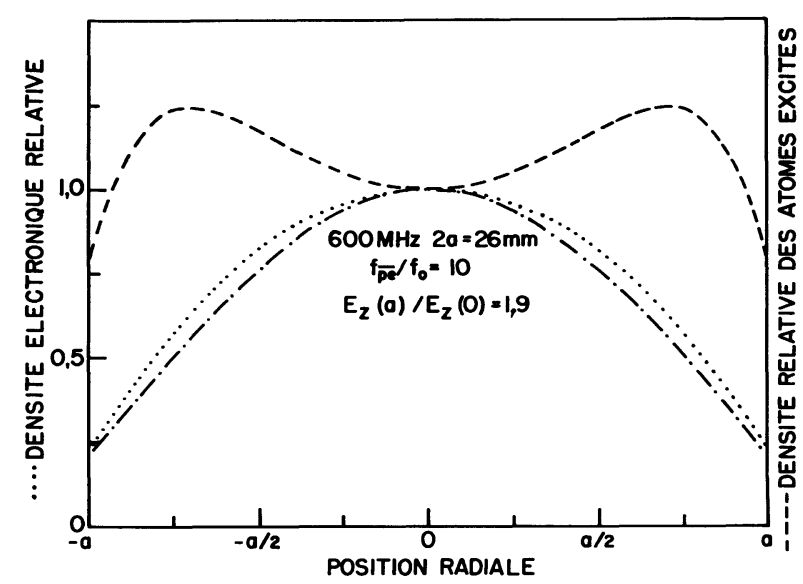

Fig. 17. - Distributions radiales de la densité électronique (pointillé) et de la densité des atomes radiatifs (traits discontinus) selon le modèle théorique. La courbe en trait-point représente la densité électronique lorsque le champ électrique est constant radialement (colonne positive).

[Theoretical radial distribution of electron density (dotted line) and of excited atoms (dashed line). The dot-dash curve represents the theoretical radial electron density distribution in a radially constant electric field (positive column).]

4.2 Distribution RADIALE DE LA DENSITÉ DES ATOMES EXCITÉS. - 4.2.1 Niveaux radiatifs. - L'évolution de la population $n_{\mathrm{j}}(r)$ d'un niveau excité radiatif s'écrit, à l'équilibre :

$$
\frac{\partial n_{\mathbf{j}}}{\partial t}=v_{\mathbf{j}} n-v_{\mathbf{j}}^{*} n_{\mathbf{j}}=0
$$

où $v_{\mathrm{j}}$ est la fréquence d'excitation du niveau $\mathrm{j}$ que nous supposerons résulter d'un processus par collisions électroniques directement à partir du fondamental et $v_{\mathbf{j}}^{*}$ est la fréquence de destruction radiative : celle-ci ne dépend pas de $r$. Compte tenu de (16), la densité d'un niveau radiatif s'écrit donc, à une constante près :

$$
n_{\mathrm{j}}(r) \simeq n(r) E^{2}(r)
$$


On considère le cas de fréquences radiatives suffisamment élevées pour que les autres mécanismes de pertes soient négligeables.

L'intensité $I_{\lambda}$ d'une raie de longueur d'onde $\lambda$ est proportionnelle à la population du niveau radiatif considéré et à sa probabilité d'émission radiative, $A(\lambda)$, ce qui, en tenant compte de la relation (19) donne :

$$
I_{\lambda} \propto n_{\mathrm{j}} \frac{A(\lambda)}{\lambda} \propto \frac{n(r) E^{2}(r) A(\lambda)}{v_{\mathbf{j}}^{*} \lambda}
$$

i.e. finalement

$$
I_{\lambda}(r) \propto n(r) E^{2}(r)
$$

4.2.2 Niveaux métastables. - Le cas des niveaux métastables est plus complexe. Le temps de vie de ces atomes excités est suffisamment grand pour que leur diffusion dans le plasma ne soit en général négligeable. L'équation complète de leur évolution, analogue à celle des électrons, s'écrit :

$$
\frac{\partial n_{\mathrm{M}}}{\partial t}=v_{\mathrm{M}} n+D_{\mathrm{M}} \nabla^{2} n_{\mathrm{M}}-n_{\mathrm{M}}\left(C_{\mathrm{M}}^{\mathrm{e}} n+k_{\mathrm{t}} n_{\mathrm{x}}\right)
$$

où $v_{M}$ est la fréquence de peuplement du niveau métastable par collision électronique, $D_{\mathrm{M}}$, le coefficient de diffusion de ces atomes métastables, $C_{\mathrm{M}}^{\mathrm{e}}$, le coefficient de destruction du niveau par collisions électroniques et $k_{\mathrm{t}}$, celui de la destruction par des atomes neutres de densité $\boldsymbol{n}_{\mathbf{x}}$.

Pour pouvoir comparer de façon simple nos résultats avec le modèle théorique, nous avons utilisé des mélanges argon-xénon et argon-azote. Dans de tels plasmas, les atomes neutres de xénon ou d'azote détruisent les atomes métastables par collisions avec une fréquence qui peut être supérieure à celle de diffusion et de destruction par les électrons. Pour un pourcentage donné de xénon ou d'azote dans l'argon, on montrera (Sect. 5.3) que le terme $k_{\mathrm{t}} n_{\mathrm{x}}$ est le terme prépondérant de pertes, ce qui fait qu'à l'état stationnaire, la relation (22) tend vers :

$$
n_{\mathrm{M}}(r) \simeq \frac{n(r) v_{\mathrm{M}}(r)}{k_{\mathrm{t}} n_{\mathrm{Xe}}}
$$

En présence du pourcentage approprié de xénon ou d'azote dans le mélange, les atomes métastables devraient donc montrer une distribution radiale de densité de même forme que celle des atomes radiatifs puisque, selon (16), $v_{\mathrm{M}}=A_{\mathrm{M}} E^{2}(r)$.

La distribution radiale théorique, dans un plasma d'onde de surface, de la densité des atomes radiatifs ou métastables, lorsque ces dernières espèces sont principalement détruites par des neutres, est représentée en traits discontinus sur la figure 17. Les calculs ont été effectués pour un champ $E_{\mathrm{T}}(r)$ donné par la relation (9) avec, comme conditions limites, $E_{\mathrm{T}}(a) / E_{\mathrm{T}}(0)=1,9$ et $n(a) / n(0)=0,2$. Ces valeurs sont typiques de celles d'une distribution expérimentale à 100 mtorr (cf. Fig. 19). On constate sur la figure 17 que c'est le profil du champ électrique qui particularise la distribution radiale des états excités dans un plasma d'onde de surface. Dans une colonne positive, le champ électrique est uniforme radialement et la distribution des états excités suit celle des électrons.

5. Comparaison des résultats avec le modèle théorique. - 5.1 Distribution RADIALE DES ÉLECTRONS. Le modèle que nous avons élaboré à la section 4 montre que l'intensité d'émission d'un niveau radiatif est proportionnelle à $n(r) E^{2}(r)$ et que d'autre part, $n(r)$ résulte de la solution du système différentiel

$$
\frac{\mathrm{d}^{2} n}{\mathrm{~d} r^{2}}+\frac{1}{r} \frac{\mathrm{d} n}{\mathrm{~d} r}+\frac{A_{\mathrm{i}}}{D_{\mathrm{a}}} I_{0}^{2}(\sigma r) n=0
$$

où $\mathrm{d} n / \mathrm{d} r=0$ en $r=0$ et $n(a) / n(0)=N(a)$ en $r=a$.

La relation (17) conduit, quelle que soit la valeur $\mathrm{du}$ paramètre $\sigma$, à une solution numérique pour $n(r)$ qui s'écarte fort peu de la fonction de Bessel $J_{0}(\mu r / a)$ avec $J_{0}(\mu)=N(a)$; on pourrait d'ailleurs montrer que ce résultat demeure vrai pour d'autres formes, très différentes, de dépendance radiale du champ électrique [7]. Physiquement, ceci tient au fait qu'en écrivant (16), nous supposons que les électrons disparaissent par recombinaison avec les ions sur les parois du tube ; ce mécanisme crée un gradient radial de densité électronique qui provoque un flux d'électrons de l'axe vers la paroi. Ce flux est contrôlé par un coefficient de diffusion $D_{\mathrm{a}}$ que nous supposons indépendant de la position. Dans cette hypothèse, quel que soit le taux de production des électrons le long du rayon, on peut s'attendre à unè distribution radiale d'électrons continûment décroissante, d'une forme proche de celle de la fonction de Bessel $J_{0}(\mu r / a)$. C'est parce que cette fonction $J_{0}$ constitue une bonne approximation de la solution numérique, quelle que soit la forme du champ électrique, que nous l'avons utilisée dans notre calcul des composantes $E_{z}(r)$ et $E_{\mathrm{r}}(r)$ de l'onde de surface (Sect. 2.2).

\subsection{DiSTRIBUTION RADIALE DES ATOMES RADIATIFS} - Nous avons choisi d'enregistrer la distribution radiale de la raie ArI $549,6 \mathrm{~nm}$, correspondant à la transition $6 \mathrm{~d} \rightarrow 4 \mathrm{p}$ (voir Fig. 8). Le niveau supérieur (6d) est optiquement relié au fondamental $\left(3 p^{6}\right)$ mais pas au métastable $4 \mathrm{~s}$. Il en résulte qu'une excitation par collisions électroniques, directement à partir du niveau fondamental, sera le mécanisme prépondérant. La relation (18) s'appliquera donc pour la raie ArI 549,6 nm.

En ce qui concerne la densité électronique, les deux inconnues de la relation (17) sont $\sigma$, la courbure du champ électrique de l'onde, et $N(a)$ que l'on ne peut poser égal à zéro, puisque dans le plasma d'onde de surface, la fréquence d'ionisation $v_{\mathbf{i}}(r)$ est en principe maximum sur la paroi. Comme il n'a pas été possible 
de mesurer directement $n(r)$, on utilisera la valeur de l'intensité relative d'émission sur la paroi pour fixer $N(a)$.

5.2.1 Ajustement numérique. - Les enregistrements des distributions radiales d'émission sont comparés avec la forme $n(r) I_{0}^{2}(\sigma r)$ où $n(r)$ est calculée par (17) pour la valeur de $\sigma$ choisie. Les valeurs de $\sigma$ et de $N(a)$ retenues sont celles qui permettent le meilleur lissage des points expérimentaux, particulièrement en ce qui concerne la position et la hauteur relative des maximums d'émission. Les valeurs de $\sigma$ et $N(a)$ ainsi ajustées ont une imprécision relative inférieure à $10 \%$.

Les figures 18,19 et 20 montrent le résultat de l'ajustement numérique (courbe en trait discontinu) de l'émission radiative à 100 mtorr pour des fréquences de 300,600 et $1000 \mathrm{MHz}$. La distribution radiale électronique calculée est représentée en pointillé. On constate que les courbures du champ électrique ainsi déterminées dépendent peu de la fréquence de l'onde puisque $E(a) / E(0)=I_{0}(\sigma a)$ varie seulement de 1,85 à $1,93\left(\sigma\right.$ de 1,29 à $\left.1,35 \mathrm{~cm}^{-1}\right)$. Par contre, l'ajustement conduit à des valeurs de densité relative sur la paroi, $N(a)$, qui croissent avec la fréquence puisqu'il en est ainsi de la luminosité relative près de la paroi. Dans le cas de $1000 \mathrm{MHz}$, la distribution expérimentale de l'émission n'est pas tout à fait symétrique; elle a été ajustée pour des valeurs légèrement différentes de $N(a)$, soit 0,33 à gauche et 0,37 à droite.

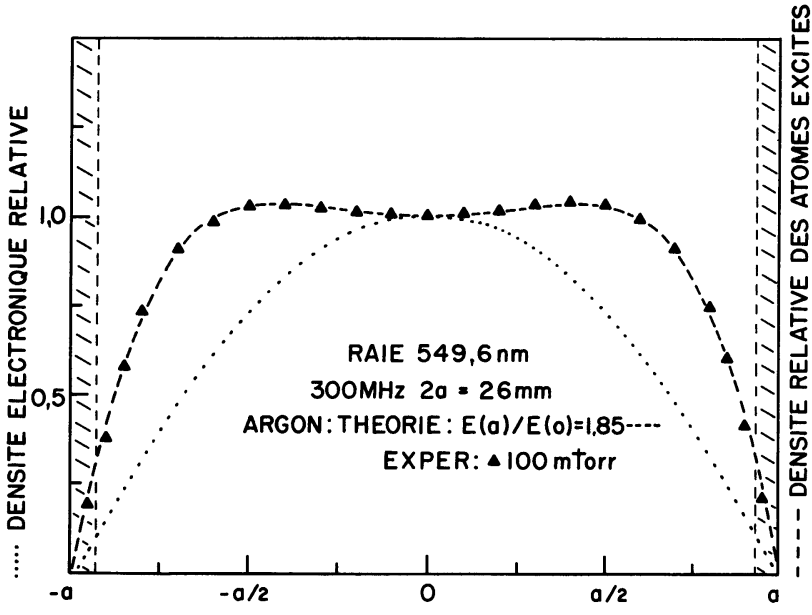

Fig. 18. - Distribution radiale de l'intensité d'émission radiative mesurée ( $(\mathbf{\Delta})$ pour $f_{0}=300 \mathrm{MHz}$ et lissage (trait discontinu) de ces points à partir de la relation $n_{\mathrm{j}}(r)=n(r) E^{2}(r)$ où $n_{\mathrm{j}}(r)$ est la densité des atomes radiatifs. Le champ $E(r)$ est approximé par la relation $I_{0}(\sigma r)$. Dans le cas présent, l'ajustement conduit à : $I_{0}(\sigma a)=E(a) / E(0)=1,85$. La densité électronique $n(r)$ esṫ représentée en pointillé.

[Comparison at $f_{0}=300 \mathrm{MHz}$ of the measured light emission $(\boldsymbol{\Delta})$ as a function of radius with a fitted theoretical curve (dashed line) based on the relation $n_{\mathrm{j}}(r)=n(r) E^{2}(r)$ representing the population of radiative atoms as a function of radius. The $E(r)$ field is approximated by $I_{0}(\sigma r)$. In the present case, the fitting yields

$$
I_{0}(\sigma a)=E(a) / E(0)=1.85 \text {. }
$$

The corresponding electron density $n(r)$ is shown as a dotted line.]

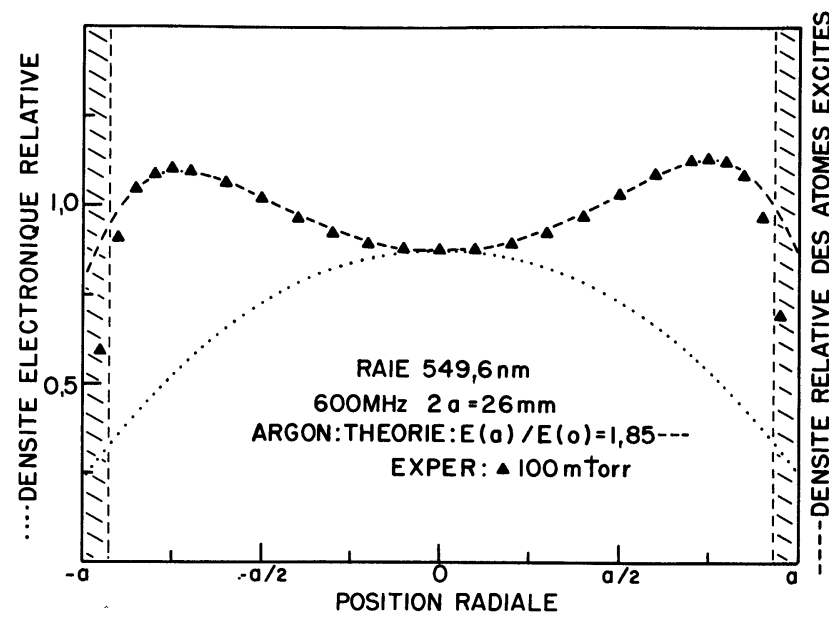

Fig. 19. - Même étude que pour la figure 18 mais avec $f_{0}=600 \mathrm{MHz}$

[As for figure 18 with $f_{0}=600 \mathrm{MHz}$.]

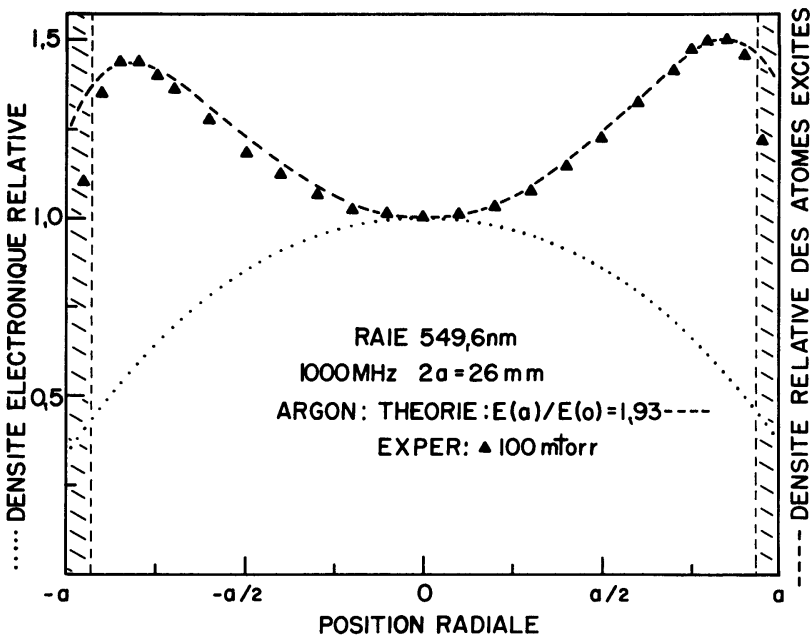

Fig. 20. - Mème étude que pour la figure 18 mall ale $f_{0}=1000 \mathrm{MHz}$

[As for figure 18 with $f_{0}=1000 \mathrm{MHz}$.]

Dans le cas du plasma de la colonne positive, le champ $E(r)$ est en principe constant, donc la distribution d'un niveau radiatif sera simplement en $n(r)$. Or, la forme de $n(r)$ dans un champ électrique constant est théoriquement en $J_{0}(\mu r / a)$. D'autre part, comme expérimentalement l'intensité lumineuse émise est nulle sur la paroi, nous posons $N(a)=0$. La figure 21 , réalisée dans une colonne positive à 50 et 100 mtorr pour la même raie $549,6 \mathrm{~nm}$, se trouve donc ajustée par une fonction de Bessel $J_{0}(2,4 r / a)$. La différence qui apparaît entre le profil théorique et l'expérience est attribuée à un phénomène de contraction [11].

5.2.2 Comparaison de la forme du champ $E_{\mathrm{T}}(r)$ déduite par ajustement avec celle calculée par les équations de l'onde de surface. - L'ajustement de $\sigma$ et $N(a)$ s'est fait pour reproduire au mieux le profil 


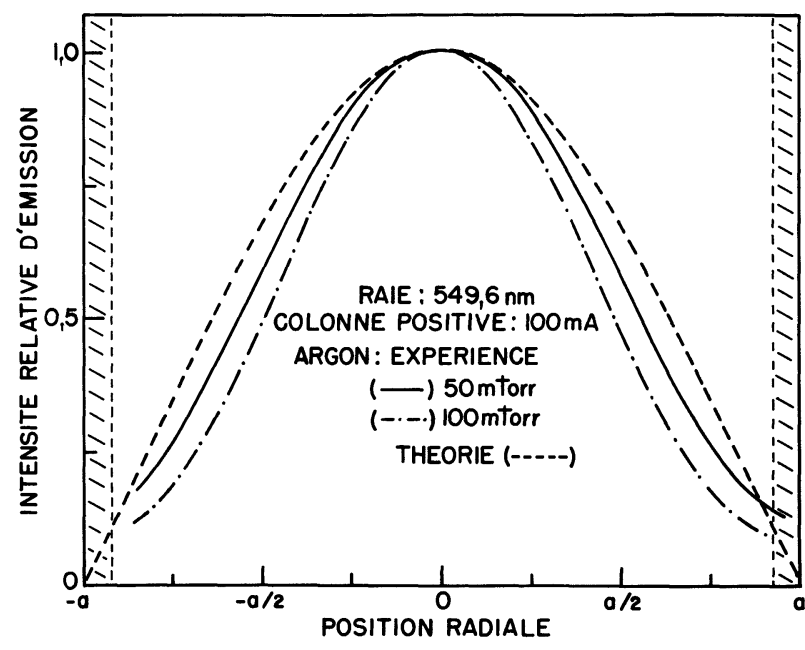

Fig. 21. - Distribution radiale de l'intensité d'émission enregistrée dans une colonne positive à $50 \mathrm{mtorr}(-)$ et $100 \mathrm{mtorr}(-.-)$ et distribution théorique correspondante (trait-discontinu).

[The radial distribution of emission intensity as measured in a positive column at 50 mtorr (-) and 100 mtorr (-. - ) and the corresponding theoretical distribution (dashed curve).]

d'émission observé. La forme du champ électrique de l'onde peut cependant être calculée à partir des équations d'onde si l'on connaît bien la densité absolue moyenne $\langle n(r)\rangle$.

En effet, si l'on suppose que $n(r)$ est de la forme $J_{0}(\mu r / a)$ et que l'on fixe $\mu$ en posant $J_{0}(\mu)=N(a)$, la connaissance de la valeur moyenne $\langle n(r)\rangle$ détermine la valeur de $\varepsilon_{\mathrm{p}}(r)$ (Eq. (6)). On peut alors parfaitement calculer $E_{z}(r)$ et $E_{\mathrm{r}}(r)$ (Eqs. (3) et (4)) et donc $E_{\mathrm{Tv}}(r)=\sqrt{E_{\mathrm{z}}^{2}(r)+E_{\mathrm{r}}^{2}(r)}$. Nous avons effectué ce calcul dans les conditions de la figure 19, en faisant varier $\langle n(r)\rangle$ comme paramètre de façon à trouver le champ $E_{\mathrm{Tv}}(r)$ qui s'approche le plus du profil $I_{0}(\sigma r)$ obtenu par l'ajustement. Comme le montre la figure 5, il existe effectivement une valeur de $\langle n(r)\rangle$ pour laquelle la forme $I_{0}(\sigma r)$ (représenté en trait discontinu) est une bonne approximation de $E_{\mathrm{Tv}}(r)$ (points). Cette valeur de $\langle n(r)\rangle$ correspond à un rapport $f_{\overline{\mathrm{pe}}} / f_{0}=2,5$. Or expérimentalement, par exemple par la décroissance de $E_{\mathrm{r}}(r)$, on trouve un rapport $f_{\overline{\mathrm{pe}}} / f_{0}$ compris entre 2,6 et 2,8 (voir Fig. 6). On conclut donc que le modèle utilisé pour expliquer le profil radial d'émission est cohérent avec les propriétés des ondes de surface et qu'il mène à des valeurs de densité électronique moyenne voisines des valeurs expérimentales.

5.3 Distribution RADIALE DES ATOMES MÉTASTABLES. - La distribution radiale des métastables $\operatorname{Ar}\left({ }^{3} \mathrm{P}_{2}\right)$ de la figure 13 , réalisée dans de l'argon pur, se trouve régie par une équation d'évolution (22) où trois processus de destruction sont susceptibles d'intervenir. L'ajustement numérique de cette distribution est possible mais il est un peu lourd. Pour faire en sorte qu'un'seul de ces termes de destruction soit important, nous avons eu recours à des mélanges contenant $10 \%$ de xénon ou $10 \%$ d'azote et $90 \%$ d'argon, en disant que dans ce cas :

$$
\begin{aligned}
k_{\mathrm{t}} n_{\mathrm{x}} & >C_{\mathrm{M}}^{\mathrm{e}} n \\
k_{\mathrm{t}} n_{\mathrm{x}} n_{\mathrm{M}} & >\left|D_{\mathrm{M}} \nabla^{2} n_{\mathrm{M}}\right|
\end{aligned}
$$

i.e. que les métastables sont détruits par collisions avec des atomes de xénon ou des molécules d'azote dans l'état fondamental. Examinons dans quelle mesure ces deux inégalités sont effectivement vérifiées. Considérons d'abord le mélange contenant $10 \%$ de xénon.

En ce qui concerne la condition (24), on sait que le coefficient $C_{\mathrm{M}}^{\mathrm{e}}$ de destruction des métastables $\operatorname{Ar}\left({ }^{3} \mathrm{P}_{2}\right)$ par collisions électroniques, déterminé dans une colonne positive, est d'environ $4( \pm 1) \times 10^{-7} \mathrm{~cm}^{3} \mathrm{~s}^{-1}$ dans l'intervalle $2<T_{\mathrm{e}}<6 \mathrm{eV}[10,12]$. Quant à la valeur du coefficient $k_{\mathrm{t}}$ représentant la destruction du ${ }^{3} \mathrm{P}_{2}$ par les neutres du xénon, elle est de

$$
18 \times 10^{-11} \mathrm{~cm}^{3} \mathrm{~s}^{-1} .
$$

(Le coefficient $k_{\mathrm{t}}$ pour la destruction $\mathrm{du}{ }^{3} \mathrm{P}_{2}$ par les neutres de l'argon est beaucoup plus faible : $0,72( \pm 0,2) \times 10^{-15} \mathrm{~cm} \mathrm{~s}^{-1}$ [10].) Dans un mélange contenant $10 \%$ de xénon, la densité $n_{\mathrm{x}}$ des neutres de xénon à 0,1 torr est de $3,6 \times 10^{14} \mathrm{~cm}^{-3}$, alors que la densité électronique moyenne mesurée à 0,1 torr et $600 \mathrm{MHz}$ est inférieure à $5 \times 10^{10} \mathrm{~cm}^{-3}$ pour un plasma de longueur $200 \mathrm{~mm}$ (voir Fig. 6). L'inégalité (24) se trouve vérifiée mais faiblement puisqu'il vient $6,5 \times 10^{4}>2 \times 10^{4}$.

Pour ce qui est de la condition (25), on peut évaluer l'ordre de grandeur de la pression minimale de xénon nécessaire à sa vérification en supposant que la diffusion des atomes métastables se fait suivant le mode fondamental de diffusion (comme pour les électrons de la colonne positive). On a :

$$
D_{\mathrm{M}} \nabla^{2} n_{\mathrm{M}}=v_{\mathrm{M}} n_{\mathrm{M}}=\frac{D_{\mathrm{M}}}{\Lambda^{2}} n_{\mathrm{M}}=\frac{\left(D_{\mathrm{M}} p\right)}{\Lambda^{2} p} n_{\mathrm{M}}
$$

où la longueur caractéristique de diffusion $\Lambda=a / 2,4$. Pour les atomes $\operatorname{Ar}\left({ }^{3} \mathrm{P}_{2}\right)$, nous prendrons comme coefficient de diffusion réduit à 1 torr,

$$
\left(D_{\mathrm{M}} p\right)=47 \mathrm{~cm}^{2} \mathrm{~s}^{-1} \text { torr }^{-1}
$$

Avec $\Lambda^{2}=0,3 \mathrm{~cm}^{2}$, pour une pression $p$ d'argon contenant $10 \%$ de xénon, on trouve

$$
p^{2}>25 \times 10^{-5} \text { torr }^{2}
$$

i.e. que l'inégalité (25) est vérifiée pour une pression minimum d'argon de $1,6 \times 10^{-3}$ torr.

Dans le cas du mélange contenant $10 \%$ d'azote, l'inégalité (24) est également satisfaite mais plus faiblement qu'avec le xénon. Ainsi à 100 mtorr, le terme de destruction par les neutres n'est que deux fois 
plus fort que celui de la destruction par les électrons. Quant à la diffusion des métastables, tout comme pour le xénon, elle est tout à fait négligeable pour 100 mtorr ou plus.

Comme nous l'avons remarqué en 4.2.2, c'est seulement si les inégalités (24) et (25) sont fortement satisfaites que $n_{\mathrm{M}}(r)$ devient proportionnelle à $n(r) E^{2}(r)$. On vient d'autre part de constater que l'inégalité (24) ne semblait pas fortement satisfaite, surtout pour le mélange $10 \%$ azote. Or, expérimentalement, les figures 14 et 16 montrent que la distribution $n_{\mathrm{M}}(r)$ est très voisine de celle $n(r) E^{2}(r)$ des atomes radiatifs émettant à $549,6 \mathrm{~nm}$ (avec néanmoins un léger décalage entre $n_{\mathrm{M}}(r)$ et $n_{\mathrm{j}}(r)$ dans le mélange $10 \%$ azote). Ceci suggère que le coefficient $C_{\mathrm{M}}^{\mathrm{e}}$ de destruction électronique possède en fait une valeur plus faible que celle que nous avons utilisée pour chiffrer la relation (24). Cette hypothèse peut être appuyée par le fait que les valeurs de $C_{\mathrm{M}}^{\mathrm{e}}$ calculées [14] sont de 2 à 4 fois plus faibles que les valeurs expérimentales trouvées $[10,12]$.

Le fait que dans le mélange à $10 \%$ de xénon, la distribution radiale de densité des métastables épouse assez bien le profil d'émission radiative (Fig. 14) permet une remarque. On pouvait en effet s'interroger sur la valeur de la mesure de l'émission radiale comparativement à celle de l'absorption comme moyen de sonder toute la longueur axiale du plasma, suivant de petits cylindres non divergents déterminés par le collimateur. Or, dans une situation où la loi radiale de l'émission et celle de la densité obtenue par absorption sont les mêmes, le fait d'observer effectivement la même dépendance radiale montre bien que la mesure du profil d'émission est significative de toute la profondeur du plasma.

5.4 REMARQUES SUR LES HYPOTHÈSES DU MODÈLE. Nous avons négligé l'existence d'une gaine sur la paroi du tube, gaine qui à faible densité électronique (300 MHz) est de l'ordre du mm. La prise en compte de cette gaine éviterait d'obtenir, lors des calculs de $E_{\mathrm{r}}(r)$, une valeur trop élevée près de la paroi.

L'hypothèse d'une température électronique indépendante du rayon, dans un plasma de pression de l'ordre du torr, en présence d'un champ HF a été utilisée avec succès [15]. D'autre part, des mesures par des sondes de Langmuir (orientées perpendiculairement à la direction du champ électrique), effectuées dans un plasma produit à $9,5 \mathrm{GHz}$, dans des conditions de produit $p a$ supérieur au nôtre, ont montré que $T_{\mathrm{e}}$, dans la limite des erreurs expérimentales, demeurait constante radialement [16]. Il est mentionné en réf. [16] que les valeurs de $T_{\mathrm{e}}$, à produits $p a$ identiques, semblent les mêmes dans les plasmas HF que dans la colonne positive, ce qui a été confirmé indirectement dans un plasma d'onde de surface par des mesures d'atténuation d'onde [2].
Le fait de poser $v_{\mathrm{j}}=A_{\mathrm{j}} E^{2}(r)$ (Eq. (16)) n'est pas entièrement nouveau. Pour expliquer l'existence d'un minimum d'intensité lumineuse au centre d'un plasma HF produit entre deux plaques parallèles la relation $v_{\mathrm{j}}=B_{\mathrm{j}} E^{2 \alpha}(r)$ a été utilisée en réf. [17] avec $B_{\mathrm{j}}$ égal à une constante et $2 \leqslant 2 \alpha \leqslant 8$. Dans le modèle proposé, $\alpha$ est un paramètre ajustable expérimentalement qui croît donc avec la pression puisque l'intensité lumineuse se creuse davantage avec elle. Dans notre cas, pour tenir compte du même phénomène, c'est le champ électrique de l'onde qui se creuse avec la pression, notamment à cause de l'augmentation de la densité électronique.

6. Conclusion. - Nous avons établi et vérifié expérimentalement un modèle prédisant la distribution radiale des atomes excités dans un plasma d'onde de surface. En particulier, nous avons montré que le profil des atomes radiatifs est de la forme $n(r) E^{2}(r)$. Il reste cependant que nous n'avons pas mesuré la distribution radiale de la densité électronique et que nous ignorons donc la valeur relative que prend cette dernière sur la paroi; cette valeur intervient directement dans le calcul de la forme du champ électrique. Cette lacune est due au fait que le plasma d'onde de surface est très fortement perturbé localement dès que l'on introduit une sonde (sonde de Langmuir, antenne), ce qui empêche la réalisation de telles mesures par les méthodes habituelles.

Nous avons remarqué que si la forme de la distribution de densité électronique du plasma d'onde de surface ne semble pas devoir être très différente de celle des colonnes positives, exception faite de la valeur prise sur la paroi qui est généralement plus grande, on obtient par contre des distributions radiales d'atomes excités très différentes : plates ou avec un creux sur l'axe plus ou moins profond. On pourrait obtenir d'autres formes de distributions radiales d'atomes excités en faisant intervenir un champ magnétique axial. Celui-ci, en fonction de son intensité, transforme graduellement l'onde de surface en onde de volume [18]. Une telle possibilité pourrait intéresser le domaine des lasers et celui de la chimie dans les plasmas.

Ce modèle peut s'appliquer aux autres types de plasmas HF fonctionnant dans les mêmes conditions de pression, pourvu que l'on connaisse la forme du champ électrique dans le plasma.

Remerciements. - Nous sommes reconnaissants à MM. F. Roy, R. Lemay et R. Martel pour leur assistance technique au cours de l'expérimentation. Ce travail a pu être réalisé grâce aux bourses et missions mises à notre disposition dans le cadre du projet intégré (01-02-19) France-Québec. 


\section{Bibliographie}

[1] Moisan, M., Beaudry, C. et Leprince, P., IEEE Trans. Plasma Sci. PS-3 (1975) 55-9.

[2] Zakrzewski, Z., Moisan, M., Glaude, V. M., Beaudry, C. LePrince, P., Plasma Phys. 19 (1977) 77-83.

[3] Moisan, M., Zakrzewski, Z. et Pantel, R., J. Phys. D 12 (1979) 219-237.

[4] Moisan, M. et Ricard, A., Can. J. Phys. 55 (1977) 1010-2.

[5] Quémada, D., Ondes dans les plasmas (Paris : Hermann) 1968.

[6] Moisan, M., Beaudry, C. et Leprince, P., Phys. Lett. 50 A (1974) 125-6.

[7] Moisan, M., Pantel, R., Ricard, A., Glaude, V. M., LePrince, P. et Allis, W. P., Rapport interne, Labo. Plasma, Université de Montréal (1979).

[8] Mrtchell, A. et Zemansky, M., Resonance Radiation and Excited Atoms (Cambridge : University Press) 1971.

[9] Hasted, J. B., Physics of Atomic Collisions (London : Butterworth) 1972 , p. 441.
[10] Delcroix, J. L., Matos Ferreira, C. et Ricard, A., Principles of Laser Plasmas édité par G. Bekefi (New York : Wiley) 1976, Chap. V.

[11] Rajotte, R., Phys. Lett. 43A (1973) 47-8.

[12] Martisovits, M., Kosinar, I., Tarabex, P. et Veis, S., Proc. 12th Int. Conf. Phen. Ionized Gases (Amsterdam : North Holland) 1975, p. 59.

[13] Eluis, E. et Twiddy, N. D., J. Phys. B 2 (1969) 1366-77.

[14] Ferreira, C. M. et Delcroix, J. L., J. Physique 36 (1975) 1233-40.

[15] Frommhold, L., Biondi, M. A. and Mehr, F. J., Phys. Rev. 165 (1968) 44-52.

[16] Kalvina, I. N., Leonteva, S. M. et Shuvalova, L. G., Opt. Spectrosc. 25 (1968) 93-6.

[17] Allis, W. P., Brown, S. C. et Everhart, E., Phys. Rev. 84 (1957) 519-22.

[18] Trivelpiece, A. W., Slow-Wave Propagation in Plasma Waveguides (San Francisco Press) 1967. 